\title{
Asymptotics for Sobolev Orthogonal Polynomials for Exponential Weights
}

\author{
J. S. Geronimo, D. S. Lubinsky, and F. Marcellan
}

Abstract. Let $\lambda>0, \alpha>1$, and let $W(x)=\exp \left(-|x|^{\alpha}\right), x \in \mathbf{R}$. Let $\psi \in L_{\infty}(\mathbf{R})$ be positive on a set of positive measure. For $n \geq 1$, one may form Sobolev orthonormal polynomials $\left(q_{n}\right)$, associated with the Sobolev inner product

$$
(f, g)=\int_{\mathbf{R}} f g(\psi W)^{2}+\lambda \int_{\mathbf{R}} f^{\prime} g^{\prime} W^{2} .
$$

We establish strong asymptotics for the $\left(q_{n}\right)$ in terms of the ordinary orthonormal polynomials $\left(p_{n}\right)$ for the weight $W^{2}$, on and off the real line. More generally, we establish a close asymptotic relationship between $\left(p_{n}\right)$ and $\left(q_{n}\right)$ for exponential weights $W=\exp (-Q)$ on a real interval $I$, under mild conditions on $Q$. The method is new and will apply to many situations beyond that treated in this paper.

\section{Introduction}

Let

$$
I=(c, d)
$$

be a real interval, finite or infinite, and let $Q: I \rightarrow[0, \infty)$ be convex. We also assume that $Q(x) \rightarrow \infty$ as $x \rightarrow c+$ or $x \rightarrow d-$. The weight

$$
W=\exp (-Q)
$$

is then called an exponential weight. Provided all power moments exist, we may define orthonormal polynomials

$$
p_{n}(x)=p_{n}\left(W^{2} ; x\right), \quad n \geq 0,
$$

satisfying

$$
\int_{I} p_{n} p_{m} W^{2}=\delta_{m n}
$$

AMS classification: 42C05, 33C25.

Key words and phrases: Orthogonal polynomials, Sobolev norms, Asymptotics. 
The leading coefficient of $p_{n}$ is denoted by $\gamma_{n}=\gamma_{n}\left(W^{2}\right)$ and assumed positive:

$$
p_{n}\left(W^{2} ; x\right)=\gamma_{n}\left(W^{2}\right) x^{n}+\cdots, \quad \gamma_{n}\left(W^{2}\right)>0 .
$$

Analysis of orthonormal polynomials for exponential weights has been a major theme in orthogonal polynomials for at least the last 30 years [4], [5], [9], [12], [14], [15], [18], [24], [28], [33]. Asymptotics for $p_{n}(x)$ as the degree $n \rightarrow \infty$ have been established for large classes of exponential weights, including

$$
W_{\alpha}(x)=\exp \left(-|x|^{\alpha}\right) \quad \text { on } I=\mathbf{R},
$$

for any $\alpha>0$. The case $\alpha=2$ is the classical Hermite weight. See, for example, [14], [15], [32].

Another theme in orthogonal polynomials that is attracting much interest is that of Sobolev orthogonal polynomials $\left(q_{n}\right)$ associated with a Sobolev inner product. Let $W$ be a weight as above, and let $\psi: I \rightarrow[0, \infty)$ be measurable, and positive on a set of positive measure. Let $\lambda>0$, and define the Sobolev inner product

$$
(f, g)=\int_{I} f g \psi^{2} W^{2}+\lambda \int_{I} f^{\prime} g^{\prime} W^{2},
$$

for all functions $f, g$ for which the inner product is meaningful. Provided all monomials are integrable with respect to $W^{2}$ and $\psi^{2} W^{2}$, this inner product generates orthonormal polynomials $\left(q_{n}\right)$ satisfying

$$
\left(q_{n}, q_{m}\right)=\delta_{m n} .
$$

We shall denote the leading coefficient of $q_{n}$ by $\kappa_{n}$, so that

$$
q_{n}(x)=\kappa_{n} x^{n}+\cdots, \quad \kappa_{n}>0 .
$$

The leading coefficient $\kappa_{n}$ admits a key extremal property

$$
\kappa_{n}^{-2}=\inf \{(P, P): P \text { monic of degree } n\} .
$$

Sobolev orthogonal polynomials have found application in a number of contexts. See, for instance, the survey papers [19], [23], as well as [16]. In particular in [13], a study of Fourier series of Sobolev orthogonal polynomials was initiated for smooth functions. Legendre and Sobolev-Legendre Fourier series were compared numerically. The measures involved in the inner product satisfied a simple algebraic relation, and were close to classical (Jacobi and Legendre) measures. Consequently, the analytical properties of the Sobolev orthogonal polynomials could be determined from those of classical orthogonal polynomials. This investigation was continued in [23] for the bounded (Jacobi) case and in [20] for the unbounded Laguerre case.

For more general measures with bounded support, a key contribution was given by Martinez-Finkelshtein [22]. There the support of the measures is a $C^{2+}$ Jordan curve, and the main requirement is that the measure in the derivative term satisfies a Szegó condition, while the other measure is arbitrary. Only the derivative part of the inner product has a significant impact on the asymptotic behavior of the Sobolev orthogonal 
polynomials. Indeed $q_{n}^{\prime}$ behaves very much like a multiple of $p_{n-1}$ in the exterior domain of the curve. If the first measure also belongs to the Szegó class, then there are relative asymptotics for $q_{n}$ in terms of $p_{n}$ in the exterior domain of the curve.

In the unbounded case, the first example, different from the Laguerre case, was considered in [1], with

$$
W(x)=\exp \left(-\frac{1}{2} x^{2}\right) \quad \text { and } \quad \psi(x)=\sqrt{x^{2}+\zeta^{2}} \quad \text { on } \mathbf{R} .
$$

Ratio asymptotics were given for $q_{n}$ in terms of the Hermite polynomials. An interesting feature is that the asymptotics depend on the parameter $\lambda$, a notable difference from the bounded case. For

$$
W(x)=\exp \left(-\frac{1}{2} x^{4}\right) \quad \text { and } \quad \psi(x)=1 \quad \text { on } \mathbf{R},
$$

a similar approach was taken in [3], using known asymptotics for Freud orthogonal polynomials.

In this paper, we shall see that $q_{n}^{\prime}$ behaves like $(1 / \sqrt{\lambda}) p_{n-1}$ for fairly general weights on an unbounded interval, but some growth restriction on $\psi$ is necessary. If $\psi$ grows too fast at $\infty$, then the first term in the inner product will swamp the derivative term. Our first result involves Freud weights, and for these, we need to define the MhaskarRakhmanov-Saff number [25], [26], [31]: for $Q$ even and convex on $\mathbf{R}$, and for $n \geq 1$, we let $a_{n}$ denote the positive root of the equation

$$
n=\frac{2}{\pi} \int_{0}^{1} a_{n} t Q^{\prime}\left(a_{n} t\right) \frac{d t}{\sqrt{1-t^{2}}} .
$$

For example, if $Q(x)=|x|^{\alpha}$, then

$$
a_{n}=C n^{1 / \alpha}, \quad n \geq 1,
$$

where the constant $C$ may be expressed in terms of gamma functions. We note that in all the cases that we consider,

$$
\lim _{n \rightarrow \infty} \frac{a_{n}}{n}=0
$$

We also need the conformal map

$$
\varphi(z)=z+\sqrt{z^{2}-1}, \quad z \in \mathbf{C} \backslash[-1,1],
$$

of the exterior of $[-1,1]$ onto the exterior of the unit disk, and the Szegó function [34] of a measurable function $f:[-\pi, \pi] \rightarrow[0, \infty)$ satisfying Szegô's condition

$$
\int_{-\pi}^{\pi} \log f(\theta) d \theta>-\infty
$$

It is defined by

$$
D(f ; z)=\exp \left(\frac{1}{4 \pi} \int_{-\pi}^{\pi} \log f\left(e^{i t}\right) \frac{e^{i t}+z}{e^{i t}-z} d t\right), \quad|z|<1,
$$


and has boundary values on the unit circle that satisfy

$$
\left|D\left(f ; e^{i \theta}\right)\right|^{2}=f(\theta) \quad \text { a.e. } \theta \in[-\pi, \pi] .
$$

We shall also need the argument of $D\left(f ; e^{i \theta}\right)$ on the unit circle. We write (whenever meaningful),

$$
D\left(f ; e^{i \theta}\right)=f(\theta)^{1 / 2} \exp (i \Gamma(f ; \theta)) .
$$

An explicit representation for $\Gamma(f ; \theta)$ is

$$
\Gamma(f ; \theta)=\frac{1}{4 \pi} P V \int_{-\pi}^{\pi} \log f(t) \cot \left(\frac{\theta-t}{2}\right) d t,
$$

where $P V$ denotes the Cauchy principal value integral.

Theorem 1.1. Let $Q: \mathbf{R} \rightarrow \mathbf{R}$ be even and continuous in $\mathbf{R}$. Assume that $Q^{\prime \prime}$ is continuous in $(0, \infty)$, and $Q^{\prime}>0$ in $(0, \infty)$. Assume, furthermore, that for some $\alpha, \beta>$ 0 ,

$$
\alpha \leq \frac{x Q^{\prime \prime}(x)}{Q^{\prime}(x)} \leq \beta, \quad x \in(0, \infty) .
$$

Let $W=e^{-Q}$, and let $\left(p_{n}\right)$ denote the orthonormal polynomials for $W^{2}$. Let $\lambda>0$ and $\psi \in L_{\infty}(\mathbf{R})$ be positive on a set of positive measure. Let $\left(q_{n}\right)$ denote the orthonormal polynomials associated with the Sobolev inner product (1). Then, as $n \rightarrow \infty$,

(I)

$$
\left\|\left(q_{n}^{\prime}-\frac{1}{\sqrt{\lambda}} p_{n-1}\right) W\right\|_{L_{2}(\mathbf{R})}=O\left(\frac{a_{n}}{n}\right)=o(1)
$$

and

$$
\left\|\left(1+\left|Q^{\prime}\right|\right)\left(q_{n}-\frac{1}{\sqrt{\lambda}} \int_{0}^{x} p_{n-1}\right) W\right\|_{L_{2}(\mathbf{R})}=O\left(\sqrt{\frac{a_{n}}{n}}\right) .
$$

$$
\left\|\left(q_{n}^{\prime}-\frac{1}{\sqrt{\lambda}} p_{n-1}\right) W\right\|_{L_{\infty}(\mathbf{R})}=O\left(\sqrt{\frac{a_{n}}{n}}\right)
$$

and

$$
\left\|\left(1+\left|Q^{\prime}\right|\right)\left(q_{n}-\frac{1}{\sqrt{\lambda}} \int_{0}^{x} p_{n-1}\right) W\right\|_{L_{\infty}(\mathbf{R})}=O\left(\sqrt{\frac{a_{n}}{n}}\right) .
$$

(III) Let

$$
W_{n}(\theta)=W\left(a_{n} \cos \theta\right), \quad \theta \in[-\pi, \pi] .
$$

Uniformly in closed subsets of $\mathbf{C} \backslash[-1,1]$,

$$
\left|\frac{\left(q_{n}^{\prime}-(1 / \sqrt{\lambda}) p_{n-1}\right)\left(a_{n} z\right)}{\varphi(z)^{n-1} D^{-2}\left(W_{n} ; 1 / \varphi(z)\right)}\right|=O\left(\frac{\sqrt{a_{n}}}{n}\right) .
$$


Uniformly in closed subsets of $\mathbf{C} \backslash[-1,1]$,

$$
\left|\frac{\left(q_{n}\left(a_{n} z\right)-(1 / \sqrt{\lambda}) \int_{0}^{a_{n} z} p_{n-1}\right)}{\varphi(z)^{n} D^{-2}\left(W_{n} ; 1 / \varphi(z)\right)}\right|=O\left(\frac{a_{n}^{3 / 2}}{n^{2}}\right) .
$$

Remarks. (a) Condition (6) allows

$$
Q(x)=|x|^{\alpha}
$$

if $\alpha>1$.

(b) What is surprising is the degree of closeness of $q_{n}^{\prime}$ to $(1 / \sqrt{\lambda}) p_{n-1}$. The $L_{2}$ asymptotics are sufficiently strong to imply the uniform bound in (II) with the aid of a simple Nikolskii inequality.

(c) The result holds assuming less smoothness of $Q$, namely for Freud weights in the class $\mathcal{F}$ (Dini), defined in [15]. Likewise the corollary below holds for Freud weights in the class $\mathcal{F}\left(\operatorname{lip} \frac{1}{2}\right)$, defined in [15]. One may also allow non-Freud $Q$, the chief requirement being that, for each $\varepsilon>0$,

$$
\frac{x Q^{\prime}(x)}{Q(x)}=O\left(Q(x)^{\varepsilon}\right)
$$

as $x$ approaches the endpoints of the interval of orthogonality. However, the formulations become more technical, so we omit them.

(d) We note that one can replace $\int_{0}^{x} p_{n-1}$ by $p_{n} \gamma_{n-1}\left(W^{2}\right) /\left(n \gamma_{n}\left(W^{2}\right)\right)$ in (8), but with a worse error term. This may be achieved with the aid of Lemma 2.3 below.

(e) We shall prove Theorem 1.1 in Section 5. Because of known asymptotics for $\left(p_{n}\right)$ [15] on and off the real line, we can deduce:

\section{Corollary 1.2.}

(a) As $n \rightarrow \infty$,

$$
\kappa_{n}=\frac{1}{n} \frac{1}{\sqrt{2 \pi \lambda}}\left(\frac{a_{n}}{2}\right)^{-n+1 / 2} \exp \left(\frac{2}{\pi} \int_{0}^{1} \frac{Q\left(a_{n} s\right)}{\sqrt{1-s^{2}}} d s\right)(1+o(1)) .
$$

(b) We have, as $n \rightarrow \infty$,

$$
\begin{aligned}
\int_{-1}^{1} \mid \sqrt{\lambda a_{n}} & q_{n}^{\prime}\left(a_{n} x\right) W\left(a_{n} x\right)-\frac{\sqrt{2 / \pi}}{\left(1-x^{2}\right)^{1 / 4}} \\
& \times\left.\cos \left[\left(n-\frac{1}{2}\right) \arccos x+2 \Gamma\left(W_{n} ; \arccos x\right)-\frac{\pi}{4}\right]\right|^{2} d x=o(1) .
\end{aligned}
$$

(c) Uniformly for $z$ in closed subsets of $\mathbf{C} \backslash[-1,1]$, we have, as $n \rightarrow \infty$,

$$
\begin{gathered}
\sqrt{\lambda a_{n}} q_{n}^{\prime}\left(a_{n} z\right) /\left\{\varphi(z)^{n-1} D^{-2}\left(W_{n} ; \frac{1}{\varphi(z)}\right)\left(1-\varphi(z)^{-2}\right)^{-1 / 2}\right\} \\
=\frac{1}{\sqrt{\pi}}(1+o(1)) .
\end{gathered}
$$


(d) There exists $\eta>0$ such that as $n \rightarrow \infty$, we have, uniformly for $|x| \leq 1-n^{-\eta}, x=$ $\cos \theta$,

$$
\begin{aligned}
& \sqrt{\lambda a_{n}} q_{n}^{\prime}\left(a_{n} x\right) W\left(a_{n} x\right)\left(1-x^{2}\right)^{1 / 4} \\
& =\sqrt{\frac{2}{\pi}} \cos \left(\left(n-\frac{1}{2}\right) \theta+2 \Gamma\left(W_{n} ; \theta\right)-\frac{\pi}{4}\right)+O\left(n^{-\eta}\right) .
\end{aligned}
$$

We note that for $\exp \left(-|x|^{\alpha}\right), \alpha>1$, more precise asymptotics follow from the results of Kriecherbauer and McLaughlin [14]. For example, one can give asymptotics for $q_{n}^{\prime}$ even around the endpoints $\pm a_{n}$ of the Mhaskar-Rakhmanov-Saff interval.

For a fairly general class of weights, for which asymptotics for the polynomials $\left(p_{n}\right)$ have not been established, we can at least prove that $q_{n}^{\prime}$ behaves like $(1 / \sqrt{\lambda}) p_{n-1}$. To state part of the asymptotics, we need the Mhaskar-Rakhmanov-Saff numbers for a nonsymmetric interval or non-even $Q$, generalizing that above [15, p. 13], [33, Theorem 1.11, p. 201]. For $Q$ convex on a (not necessarily symmetric) interval $I$, there are unique numbers $a_{ \pm n}$, the so-called Mhaskar-Rakhmanov-Saff numbers $a_{ \pm n}$, satisfying

$$
c<a_{-n}<0<a_{n}<d
$$

and

$$
\begin{aligned}
& n=\frac{1}{\pi} \int_{a_{-n}}^{a_{n}} \frac{x Q^{\prime}(x)}{\sqrt{\left(x-a_{-n}\right)\left(a_{n}-x\right)}} d x, \\
& 0=\frac{1}{\pi} \int_{a_{-n}}^{a_{n}} \frac{Q^{\prime}(x)}{\sqrt{\left(x-a_{-n}\right)\left(a_{n}-x\right)}} d x .
\end{aligned}
$$

We also let

$$
\delta_{n}=\frac{1}{2}\left(a_{n}+\left|a_{-n}\right|\right),
$$

and let $L_{n}$ denote the linear map of $\left[a_{-n}, a_{n}\right]$ onto $[-1,1]$ so that

$$
L_{n}(z)=-1+\frac{z-a_{-n}}{\delta_{n}} .
$$

Its inverse linear map is denoted $L_{n}^{[-1]}$. Amongst the properties of $a_{ \pm n}$ is the Mhaskar-Saff identity [24], [25], [26], [33],

$$
\|P W\|_{L_{\infty}(I)}=\|P W\|_{L_{\infty}\left[a_{-n}, a_{n}\right]},
$$

valid for polynomials $P$ of degree $\leq n$.

Theorem 1.3. Let $I=(c, d)$ be a finite or infinite interval containing 0 . Let $Q$ : $I \rightarrow[0, \infty)$ and let $Q^{\prime}$ be an increasing absolutely continuous function in I, with $Q^{\prime}(0)=0=Q(0)$ and $Q$ positive in $I \backslash\{0\}$. Assume also that, for $j=0,1$,

$$
\lim _{x \rightarrow c+}\left|Q^{(j)}(x)\right|=\infty=\lim _{x \rightarrow d-}\left|Q^{(j)}(x)\right| .
$$


Let $W=e^{-Q}$, and let $\left(p_{n}\right)$ denote the orthonormal polynomials for $W^{2}$. Let $\lambda>0$, $\psi \in L_{\infty}(\mathbf{R})$ be positive on a set of positive measure, and let $\left(q_{n}\right)$ denote the orthonormal polynomials associated with the Sobolev inner product (1). Then

(I)

$$
\lim _{n \rightarrow \infty}\left\|\left(q_{n}^{\prime}-\frac{1}{\sqrt{\lambda}} p_{n-1}\right) W\right\|_{L_{2}(I)}=0 .
$$

If we assume in addition that

$$
\limsup _{x \rightarrow c+\text { or } x \rightarrow d-} \frac{Q^{\prime \prime}(x)}{Q^{\prime}(x)^{2}}<1,
$$

then

$$
\lim _{n \rightarrow \infty}\left\|\left(1+\left|Q^{\prime}\right|\right)\left(q_{n}-q_{n}(0)-\frac{1}{\sqrt{\lambda}} \int_{0}^{x} p_{n-1}\right) W\right\|_{L_{2}(I)}=0 .
$$

(II) Let

$$
W_{n}(\theta)=W\left(L_{n}^{[-1]}(\cos \theta)\right), \quad \theta \in[-\pi, \pi] .
$$

Uniformly in closed subsets of $\mathbf{C} \backslash[-1,1]$,

$$
\lim _{n \rightarrow \infty}\left|\frac{\delta_{n}^{1 / 2}\left(q_{n}^{\prime}-(1 / \sqrt{\lambda}) p_{n-1}\right)\left(L_{n}^{[-1]}(z)\right)}{\varphi(z)^{n-1} D^{-2}\left(W_{n} ; 1 / \varphi(z)\right)}\right|=0 .
$$

Let

$$
W_{n}^{*}(\theta)=\left(1+\left|Q^{\prime}\left(L_{n}^{[-1]}(\cos \theta)\right)\right|\right) W_{n}(\theta), \quad \theta \in[-\pi, \pi] .
$$

If we also assume (22) then, uniformly in closed subsets of $\mathbf{C} \backslash[-1,1]$,

$$
\lim _{n \rightarrow \infty}\left|\frac{\delta_{n}^{1 / 2}\left(q_{n}\left(L_{n}(z)\right)-q_{n}(0)-(1 / \sqrt{\lambda}) \int_{0}^{L_{n}(z)} p_{n-1}\right)}{\varphi(z)^{n} D^{-2}\left(W_{n}^{*} ; 1 / \varphi(z)\right)}\right|=0 .
$$

Remarks. (a) Note that since $Q^{\prime}$ grows to infinity as we approach the endpoints of $I$, (23) actually gives a rate of convergence for the limit.

(b) The restriction (22) is a regularity condition, rather than a growth one. If we do not assume it, we can still prove a version of (23) in which $\left|Q^{\prime}\right|$ is replaced by a function that grows more slowly as we approach the endpoints of $I$.

One of our main tools is an estimate relating the leading coefficient $\kappa_{n}$ of $q_{n}$ to the leading coefficient $\gamma_{n-1}\left(W^{2}\right)$ of $p_{n-1}\left(W^{2} ; x\right)$. Its formulation involves the weighted $L_{2}$ error of approximation

$$
E_{n}[f ; W]=\inf \left\{\|(f-P) W\|_{L_{2}(I)}: \operatorname{deg}(P) \leq n\right\}
$$

and the linear operator

$$
I[R](x)=W(x)^{-2} \int_{c}^{x} R(t) W^{2}(t) \psi^{2}(t) d t, \quad x \in(c, d),
$$

defined on suitably restricted classes of functions $R$. We prove: 
Theorem 1.4. Let I be a finite or infinite interval, and let $W: I \rightarrow \mathbf{R}$ be a measurable function such that $W^{2}$ has all finite power moments and corresponding orthonormal polynomials $\left(p_{n}\right)$. Let $\psi: I \rightarrow \mathbf{R}$ be a measurable function such that $(\psi W)^{2}$ has all finite power moments, and assume that $\psi$ is positive on a set of positive measure. Let $\lambda>0$, and let $\left(q_{n}\right)$ denote the orthonormal polynomials associated with the inner product (1), with leading coefficients $\left(\kappa_{n}\right)$. Then

$$
\begin{aligned}
0 & \leq\left(\frac{\gamma_{n-1}\left(W^{2}\right)}{n \kappa_{n}}\right)^{2}-\left\{\left(\frac{\gamma_{n-1}\left(W^{2}\right)}{n \gamma_{n}\left((\psi W)^{2}\right)}\right)^{2}+\lambda\right\} \\
& \leq \sup E_{n-2}^{2}[I[R] ; W],
\end{aligned}
$$

where the sup is taken over all polynomials $R$ of degree $\leq n-1$ satisfying both

$$
\|R W \psi\|_{L_{2}(I)}=1
$$

and

$$
\int_{I} R(W \psi)^{2}=0
$$

To estimate the error in approximation, one needs a Jackson-type estimate. This reduces the right-hand side in (30) to a bound on the weighted derivative $I[R]^{\prime}$, and standard methods enable one to prove weighted boundedness of $I[R]^{\prime}$ at least for exponential weights.

Theorem 1.5. Let $I=(c, d)$ be a finite or infinite interval containing 0 . Let $Q$ : $I \rightarrow[0, \infty)$ and let $Q^{\prime}$ be an increasing absolutely continuous function in $I$, with $Q^{\prime}(0)=0=Q(0)$ and $Q$ positive in $I \backslash\{0\}$. Let $\lambda>0$ and let $\psi \in L_{\infty}(\mathbf{R})$ be positive on a set of positive measure. Assume also that there exists a sequence of positive numbers $\left(\eta_{n}\right)$ such that $W$ admits the Jackson estimate

$$
E_{n}[f ; W] \leq \eta_{n}\left\|f^{\prime} W\right\|_{L_{2}(I)}
$$

for all $n \geq 1$ and absolutely continuous functions $f: I \rightarrow \mathbf{R}$. Then

$$
\left|\left(\frac{\gamma_{n-1}\left(W^{2}\right)}{n \kappa_{n}}\right)^{2}-\lambda\right| \leq\left(\|\psi\|_{L_{\infty}(I)} \frac{\gamma_{n-1}\left(W^{2}\right)}{n \gamma_{n}\left(W^{2}\right)}\right)^{2}+C_{1} \eta_{n-2}^{2}
$$

The constant $C_{1}$ is independent of $n, \lambda$ but depends on $W, \psi$.

Under the conditions on $Q$ in Theorem 1.3, we shall establish the Jackson estimate (33) in Section 3. We now show that some restriction on the growth of $\psi$ is necessary near the endpoints of $I$.

Example 1.6. In Theorems 1.1 and 1.3, we assumed that $\psi$ is bounded. We now show by example, that if $\psi$ grows faster than $\sqrt{Q^{\prime}}$ near the endpoints of $I$, then the limit (21) can fail. Observe that

$$
q_{n}^{\prime}-\frac{1}{\sqrt{\lambda}} p_{n-1}=\left[\frac{n \kappa_{n}}{\gamma_{n-1}\left(W^{2}\right)}-\frac{1}{\sqrt{\lambda}}\right] p_{n-1}+S
$$


where $S$ has degree $\leq n-2$, and hence

$$
\int_{I}\left(q_{n}^{\prime}-\frac{1}{\sqrt{\lambda}} p_{n-1}(x)\right)^{2} W^{2} \geq\left[\frac{n \kappa_{n}}{\gamma_{n-1}\left(W^{2}\right)}-\frac{1}{\sqrt{\lambda}}\right]^{2} .
$$

Let $W$ be the Hermite weight

$$
W(x)=\exp \left(-\frac{1}{2} x^{2}\right), \quad x \in \mathbf{R},
$$

let $\Delta>1$, and let

$$
\psi(x)=|x|^{\Delta}, \quad x \in \mathbf{R} .
$$

We shall show that

$$
\lim _{n \rightarrow \infty, n \text { even }} \frac{n \kappa_{n}}{\gamma_{n-1}\left(W^{2}\right)}=0
$$

so (35) gives

$$
\liminf _{n \rightarrow \infty, n \text { even }} \int_{I}\left(q_{n}^{\prime}-\frac{1}{\sqrt{\lambda}} p_{n-1}(x)\right)^{2} W^{2} \geq \frac{1}{\lambda}>0,
$$

so (21) fails. By the left inequality in (30),

$$
\frac{n \kappa_{n}}{\gamma_{n-1}\left(W^{2}\right)} \leq \frac{n \gamma_{n}\left((\psi W)^{2}\right)}{\gamma_{n-1}\left(W^{2}\right)} .
$$

We can use explicit formulas for the leading coefficients in this last right-hand side to show that it decays to 0. First, [34, pp. 105-106],

$$
\gamma_{n}\left(W^{2}\right)=\pi^{-1 / 4}\left(2^{n} / n !\right)^{1 / 2} .
$$

Next,

$$
(\psi W)^{2}(x)=|x|^{2 \Delta} \exp \left(-x^{2}\right)
$$

is the symmetrized form of the Laguerre weight $t^{-1 / 2+\Delta} \exp (-t)$, under the transformation $t=x^{2}$ in the integral defining orthonormality. Representations for Laguerre polynomials [34, pp. 100-101] give, for $n=2 m$,

$$
\gamma_{n}\left((\psi W)^{2}\right)=\left\{\frac{1}{\Gamma\left(\frac{1}{2}+\Delta\right)} \frac{1}{\left(\begin{array}{c}
\left.m+\Delta-\frac{1}{2}\right) \\
m
\end{array}\right)}\right\}^{1 / 2} \frac{1}{m !} .
$$

Substituting these representations into (36) and applying Stirling's formula, gives, for even $n$,

$$
\frac{n \kappa_{n}}{\gamma_{n-1}\left(W^{2}\right)} \leq C\left(n^{1-\Delta}\right)^{1 / 2}
$$

This decays to 0 if $\Delta>1$. 
At least for Freud weights, it seems likely that (21) will persist if we ensure that

$$
\lim _{|x| \rightarrow \infty} \psi^{2}(x) / Q^{\prime}(x)=0 .
$$

However the growth of $\psi$ affects the error term in (7), and we no longer necessarily obtain the uniform asymptotic (9).

We note finally that in the limit case $\Delta=1$, the results of [1] imply that there exist $C_{1}, C_{2}>0$ such that, for $n$ large enough and for all $\lambda>0$,

$$
C_{1} / \sqrt{\varphi(1+2 \lambda)} \leq \frac{n \kappa_{n}}{\gamma_{n-1}\left(W^{2}\right)} \leq C_{2} / \sqrt{\varphi(1+2 \lambda)}
$$

so the ratio does not decay to 0 .

As a final example in this section, we apply Theorem 1.1 to orthonormal expansions.

Application 1.7. Let $W=\exp (-Q)$ be as in Theorem 1.1 and let $f: \mathbf{R} \rightarrow \mathbf{R}$ be differentiable a.e. with $(f, f)$ finite. Assume for simplicity that $\psi \equiv 1$. We may then form the Sobolev orthonormal expansion

$$
\sum_{n=0}^{\infty}\left(f, q_{n}\right) q_{n}
$$

We shall show that there is a close relationship between the term-by-term derivative of this series, namely,

$$
\sum_{n=0}^{\infty}\left(f, q_{n}\right) q_{n}^{\prime}
$$

and the standard orthonormal expansion of $f^{\prime}$ in $\left(p_{n}\right)$. More precisely, we shall show that

$$
\begin{gathered}
\left\|\left[\sum_{n=m}^{\infty}\left(f, q_{n}\right) q_{n}^{\prime}-\sum_{n=m-1}^{\infty}\left(\int_{\mathbf{R}} f^{\prime} p_{n} W^{2}\right) p_{n}\right] W\right\|_{L_{2}(\mathbf{R})} \\
\leq C(f, f)^{1 / 2}\left(\sum_{n=m}^{\infty} \frac{a_{n}^{2}}{n^{2}}\right)^{1 / 2},
\end{gathered}
$$

where $C$ is independent of $f$ and $m$. If, for example, $Q(x)=|x|^{\alpha}$, where $\alpha>2$, then this series converges, and the right-hand side of (37) is $O\left(n^{1 / \alpha-1 / 2}\right)$. To establish (37), we write

$$
\left(f, q_{n}\right) q_{n}^{\prime}=\left(f, q_{n}\right)\left[q_{n}^{\prime}-\frac{1}{\sqrt{\lambda}} p_{n-1}\right]+\frac{1}{\sqrt{\lambda}}\left(f, q_{n}\right) p_{n-1}
$$

and

$$
\begin{aligned}
\frac{1}{\sqrt{\lambda}}\left(f, q_{n}\right) p_{n-1}= & \frac{1}{\sqrt{\lambda}}\left(\int_{\mathbf{R}} f q_{n} W^{2}\right) p_{n-1} \\
& +\sqrt{\lambda}\left(\int_{\mathbf{R}} f^{\prime}\left[q_{n}^{\prime}-\frac{1}{\sqrt{\lambda}} p_{n-1}\right] W^{2}\right) p_{n-1} \\
& +\left(\int_{\mathbf{R}} f^{\prime} p_{n-1} W^{2}\right) p_{n-1} .
\end{aligned}
$$


Using these last two identities, we see that the left-hand side in (37) is bounded above by

$$
\begin{aligned}
\sum_{n=m}^{\infty}\left|\left(f, q_{n}\right)\right| & \left\|\left[q_{n}^{\prime}-\frac{1}{\sqrt{\lambda}} p_{n-1}\right] W\right\|_{L_{2}(\mathbf{R})}+\frac{1}{\sqrt{\lambda}}\left(\sum_{n=m}^{\infty}\left[\int_{\mathbf{R}} f q_{n} W^{2}\right]^{2}\right)^{1 / 2} \\
& +\sqrt{\lambda}\left(\sum_{n=m}^{\infty}\left[\int_{\mathbf{R}} f^{\prime}\left[q_{n}^{\prime}-\frac{1}{\sqrt{\lambda}} p_{n-1}\right] W^{2}\right]^{2}\right)^{1 / 2}=: T_{1}+T_{2}+T_{3} .
\end{aligned}
$$

Here, by (7), the triangle inequality, and Bessel's inequality,

$$
\begin{aligned}
T_{1} & \leq C\left(\sum_{n=m}^{\infty}\left|\left(f, q_{n}\right)\right|^{2}\right)^{1 / 2}\left(\sum_{n=m}^{\infty} \frac{a_{n}^{2}}{n^{2}}\right)^{1 / 2} \\
& \leq C(f, f)^{1 / 2}\left(\sum_{n=m}^{\infty} \frac{a_{n}^{2}}{n^{2}}\right)^{1 / 2} .
\end{aligned}
$$

Next,

$$
\left[\int_{\mathbf{R}} f q_{n} W^{2}\right]^{2} \leq\left[\int_{\mathbf{R}} f^{2} W^{2}\right]\left[\int_{\mathbf{R}} q_{n}^{2} W^{2}\right] \leq(f, f) C\left(\frac{a_{n}}{n}\right)^{2}
$$

see (78), (88) below. Then $T_{2}$ admits a similar estimate to $T_{1}$. Finally, as

$$
\begin{aligned}
{\left[\int_{\mathbf{R}} f^{\prime}\left[q_{n}^{\prime}-\frac{1}{\sqrt{\lambda}} p_{n-1}\right] W^{2}\right]^{2} } & \leq\left[\int_{\mathbf{R}} f^{\prime 2} W^{2}\right]\left[\int_{\mathbf{R}}\left[q_{n}^{\prime}-\frac{1}{\sqrt{\lambda}} p_{n-1}\right]^{2} W^{2}\right] \\
& \leq \frac{1}{\lambda}(f, f) C\left(\frac{a_{n}}{n}\right)^{2},
\end{aligned}
$$

by (7) again, $T_{3}$ also admits an estimate similar to that for $T_{1}$. Then (37) follows.

\section{Proof of Theorems 1.4 and 1.5}

Throughout, we assume the hypotheses of Theorem 1.4. We also let $\mathcal{P}_{n}^{m}$ denote the set of monic polynomials of degree $n$.

Proof of Theorem 1.4. First,

$$
\begin{aligned}
\kappa_{n}^{-2} & =\inf _{P \in \mathcal{P}_{n}^{m}}(P, P) \\
& \geq \inf _{P \in \mathcal{P}_{n}^{m}} \int_{I} P^{2}(\psi W)^{2}+\lambda \inf _{P \in \mathcal{P}_{n}^{m}} \int_{I}\left(P^{\prime}\right)^{2} W^{2} \\
& =\gamma_{n}^{-2}\left((\psi W)^{2}\right)+\lambda n^{2} \gamma_{n-1}^{-2}\left(W^{2}\right),
\end{aligned}
$$

as $P \in \mathcal{P}_{n}^{m}$ implies that $P^{\prime} / n \in \mathcal{P}_{n-1}^{m}$, and by the extremal property of the leading coefficient of orthonormal polynomials. In the other direction, fix $a \in I$, and define a 
monic polynomial $P_{*}$ by

$$
P_{*}(x)=\frac{n}{\gamma_{n-1}\left(W^{2}\right)} \int_{a}^{x} p_{n-1}\left(W^{2} ; t\right) d t+C,
$$

where the constant of integration $C$ is chosen so that

$$
\int_{I} P_{*} p_{0}\left((\psi W)^{2}\right)(\psi W)^{2}=0
$$

Observe that

$$
\lambda \int_{I}\left(P_{*}^{\prime}\right)^{2} W^{2}=\lambda\left(\frac{n}{\gamma_{n-1}\left(W^{2}\right)}\right)^{2} .
$$

Now expand in terms of the orthonormal polynomials for the weight $(\psi W)^{2}$ :

$$
P_{*}=\sum_{j=1}^{n-1} b_{j} p_{j}\left((\psi W)^{2}\right)+\frac{1}{\gamma_{n}\left((\psi W)^{2}\right)} p_{n}\left((\psi W)^{2}\right),
$$

SO

$$
\int_{I} P_{*}^{2}(\psi W)^{2}=\sum_{j=1}^{n-1} b_{j}^{2}+\gamma_{n}^{-2}\left((\psi W)^{2}\right) .
$$

(The coefficient of $p_{0}\left((\psi W)^{2}\right)$ is zero by (39).) An integration by parts gives

$$
\begin{aligned}
b_{j} & =\int_{I} P_{*} p_{j}\left((\psi W)^{2}\right)(\psi W)^{2} \\
& =-\frac{n}{\gamma_{n-1}\left(W^{2}\right)} \int_{c}^{d} p_{n-1}\left(W^{2} ; x\right)\left\{\int_{c}^{x} p_{j}\left((\psi W)^{2} ; t\right)(\psi W)^{2}(t) d t\right\} d x .
\end{aligned}
$$

To see that the first terms in the integration by parts, namely,

$$
\left[P_{*}(x) \int_{c}^{x} p_{j}\left((\psi W)^{2} ; t\right)(\psi W)^{2}(t) d t\right]_{x=c}^{x=d},
$$

do indeed vanish, we note that it is trivial if $c, d$ are finite. Suppose now that $c=-\infty$. It suffices to show that for each pair of nonnegative integers $(k, \ell)$, we have

$$
\lim _{x \rightarrow-\infty}|x|^{k} \int_{c}^{x}|t|^{\ell}(\psi W)^{2}(t) d t=0 .
$$

The integral in this last limit is bounded above for $x<0$ by

$$
\int_{c}^{x}|t|^{\ell+k}(\psi W)^{2}(t) d t
$$

and that decays to 0 as $x \rightarrow-\infty$ by convergence of the power moments of $(\psi W)^{2}$. Similarly, we may handle the case $d=\infty$, also using that

$$
\int_{c}^{x} p_{j}\left((\psi W)^{2} ; t\right)(\psi W)^{2}(t) d t=-\int_{x}^{d} p_{j}\left((\psi W)^{2} ; t\right)(\psi W)^{2}(t) d t .
$$


So (43) is correct as stated. Next, we use the dual formulation,

$$
\left(\sum_{j=1}^{n-1} b_{j}^{2}\right)^{1 / 2}=\sup \left\{\sum_{j=1}^{n-1} c_{j} b_{j}: \sum_{j=1}^{n-1} c_{j}^{2}=1\right\} .
$$

For any such $\left\{c_{j}\right\}_{j=1}^{n-1}$, set

$$
R=\sum_{j=1}^{n-1} c_{j} p_{j}\left((\psi W)^{2}\right)
$$

so

$$
\begin{aligned}
\sum_{j=1}^{n-1} c_{j} b_{j} & =-\frac{n}{\gamma_{n-1}\left(W^{2}\right)} \int_{c}^{d} p_{n-1}\left(W^{2} ; x\right)\left\{\int_{c}^{x} R(t)(\psi W)^{2}(t) d t\right\} d x \\
& =-\frac{n}{\gamma_{n-1}\left(W^{2}\right)} \int_{c}^{d} p_{n-1}\left(W^{2} ; x\right) I[R](x) W^{2}(x) d x
\end{aligned}
$$

with the notation (29). If $S$ is any polynomial of degree $\leq n-2$, we can use orthogonality to continue this as

$$
\begin{aligned}
\sum_{j=1}^{n-1} c_{j} b_{j} & =-\frac{n}{\gamma_{n-1}\left(W^{2}\right)} \int_{c}^{d} p_{n-1}\left(W^{2} ; x\right)(I[R](x)-S(x)) W^{2}(x) d x \\
& \leq \frac{n}{\gamma_{n-1}\left(W^{2}\right)}\left\{\int_{c}^{d}(I[R](x)-S(x))^{2} W^{2}(x) d x\right\}^{1 / 2} .
\end{aligned}
$$

Since $S$ is any such polynomial, we obtain

$$
\sum_{j=1}^{n-1} c_{j} b_{j} \leq \frac{n}{\gamma_{n-1}\left(W^{2}\right)} E_{n-2}[I[R] ; W] .
$$

Finally the only restriction on $R$, apart from having degree $\leq n-1$ and that the sum of the squares of the coefficients is 1 , is that its coefficient of $p_{0}\left((\psi W)^{2}\right)$ is zero, that is,

$$
\int_{I} R p_{0}\left((\psi W)^{2}\right)(\psi W)^{2}=0
$$

Since $p_{0}$ is a constant, this reduces to (32). Hence, taking sup over all $\left(c_{j}\right)_{j=1}^{n-1}$ whose sum of squares is 1 , we obtain

$$
\left(\sum_{j=1}^{n-1} b_{j}^{2}\right)^{1 / 2} \leq \frac{n}{\gamma_{n-1}\left(W^{2}\right)} \sup E_{n-2}[I[R] ; W],
$$

where the sup is taken over all polynomials $R$ of degree $\leq n-1$ satisfying both (31) and (32). Combining this with (40) and (42) gives

$$
\begin{aligned}
\kappa_{n}^{-2} & \leq\left(P^{*}, P^{*}\right) \\
& \leq\left(\frac{n}{\gamma_{n-1}\left(W^{2}\right)}\right)^{2}\left(\sup E_{n-2}[I[R] ; W]\right)^{2}+\gamma_{n}^{-2}\left((\psi W)^{2}\right)+\lambda\left(\frac{n}{\gamma_{n-1}\left(W^{2}\right)}\right)^{2} .
\end{aligned}
$$

Rearranging this and using (38) gives the result. 
In the course of the above proof, we actually proved:

Lemma 2.1. Assume the hypotheses of Theorem 1.4. Let $a \in I, n \geq 1$, and let $b_{0}$ denote the coefficient of $p_{0}\left((\psi W)^{2} ; x\right)$ in the orthonormal expansion of the polynomial $\left(n / \gamma_{n-1}\left(W^{2}\right)\right) \int_{a}^{x} p_{n-1}\left(W^{2}\right)$ with respect to the polynomials $\left\{p_{j}\left((\psi W)^{2} ; x\right)\right\}_{j=0}^{n}$. Then

$$
\begin{aligned}
\|\left(\frac{n}{\gamma_{n-1}\left(W^{2}\right)}\right. & \int_{a}^{x} p_{n-1}\left(W^{2}\right)-b_{0} p_{0}\left((\psi W)^{2}\right) \\
& \left.-\frac{1}{\gamma_{n}\left((\psi W)^{2}\right)} p_{n}\left((\psi W)^{2}\right)\right) \psi W \|_{L_{2}(I)} \\
\leq & \frac{n}{\gamma_{n-1}\left(W^{2}\right)} \sup E_{n-2}[I[R] ; W],
\end{aligned}
$$

where the sup is taken over all polynomials $R$ of degree $\leq n-1$ satisfying both (31) and (32).

For the proof of Theorem 1.5, we need a lemma, which follows the classical ideas of Freud. It is presented in Mhaskar's book [24, p. 84 ff.] for Freud weights.

Lemma 2.2. Assume the conditions on $W$ and $\psi$ in Theorem 1.5 . Let $1 \leq p \leq \infty$. Let $h: I \rightarrow \mathbf{R}$ be measurable, with

$$
\int_{I} h(\psi W)^{2}=0
$$

Then

$$
\left\|I[h]^{\prime} W\right\|_{L_{p}(I)} \leq C_{p}\|h W \psi\|_{L_{p}(I)},
$$

where $C_{p}$ depends only on $p, W$, and $\psi$.

Proof. We prove this first for $p=\infty$, then $p=1$, and then use interpolation to do $1<p<\infty$.

Step 1: $p=\infty$.

Assume first $x \in(c, 0)$ and recall that we are assuming that $Q^{\prime}(x) \leq Q^{\prime}(0)=0$ for such $x$. Now

$$
I[h](x)=e^{2 Q(x)} \int_{c}^{x} h(t) e^{-2 Q(t)} \psi^{2}(t) d t,
$$

so, for a.e. $x \in(c, 0)$,

$$
\begin{aligned}
I[h]^{\prime}(x) & =2 Q^{\prime}(x) e^{2 Q(x)} \int_{c}^{x} h(t) e^{-2 Q(t)} \psi^{2}(t) d t+h(x) \psi^{2}(x) \\
& \Rightarrow\left|I[h]^{\prime}(x) W(x)\right| \\
& \leq\|h W \psi\|_{L_{\infty}(I)}\|\psi\|_{L_{\infty}(I)}\left\{2\left|Q^{\prime}(x)\right| e^{Q(x)} \int_{c}^{x} e^{-Q(t)} d t+1\right\} .
\end{aligned}
$$


Because $Q^{\prime}$ is increasing, and negative in $(c, 0)$,

$$
\left|Q^{\prime}(x)\right| e^{Q(x)} \int_{c}^{x} e^{-Q(t)} d t \leq e^{Q(x)}\left|\int_{c}^{x} e^{-Q(t)} Q^{\prime}(t) d t\right|=1 .
$$

So, for $x<0$,

$$
\left|I[h]^{\prime}(x) W(x)\right| \leq 3\|\psi\|_{L_{\infty}(I)}\|h W \psi\|_{L_{\infty}(I)} .
$$

Next, for $x>0$, we use (45) to deduce that

$$
I[h](x)=-e^{2 Q(x)} \int_{x}^{d} h(t) e^{-2 Q(t)} \psi^{2}(t) d t
$$

and proceed similarly. In summary,

$$
\left\|I[h]^{\prime} W\right\|_{L_{\infty}(I)} \leq 3\|\psi\|_{L_{\infty}(I)}\|h W \psi\|_{L_{\infty}(I)} .
$$

Step 2: $p=1$.

Now

$$
\begin{aligned}
\left\|I[h]^{\prime} W\right\|_{L_{1}(c, 0)}= & \int_{c}^{0}\left|I[h]^{\prime}(x) W(x)\right| d x \\
\leq & \int_{c}^{0} 2\left|Q^{\prime}\right|(x) e^{Q(x)}\left|\int_{c}^{x} h(t) e^{-2 Q(t)} \psi^{2}(t) d t\right| d x \\
& +\int_{c}^{0}|h(x)| e^{-Q(x)} \psi^{2}(x) d x \\
\leq & \|\psi\|_{L_{\infty}(I)}\left(\begin{array}{c}
2 \int_{c}^{0}|h(t)| e^{-2 Q(t)} \psi(t)\left[\int_{t}^{0}\left|Q^{\prime}\right|(x) e^{Q(x)} d x\right] d t \\
\quad+\int_{c}^{0}|h(x)| e^{-Q(x)}|\psi(x)| d x
\end{array}\right) .
\end{aligned}
$$

Here, as $Q^{\prime} \leq 0$ in $(c, 0)$, in the integral,

$$
\int_{t}^{0}\left|Q^{\prime}\right|(x) e^{Q(x)} d x=e^{Q(t)}-e^{Q(0)} \leq e^{Q(t)},
$$

so

$$
\left\|I[h]^{\prime} W\right\|_{L_{1}(c, 0)} \leq\left(3\|\psi\|_{L_{\infty}(I)}\right)\|h W \psi\|_{L_{1}(c, 0)} .
$$

Similarly, using (45), we deduce that

$$
\left\|I[h]^{\prime} W\right\|_{L_{1}(0, d)} \leq\left(3\|\psi\|_{L_{\infty}(I)}\right)\|h W \psi\|_{L_{1}(0, d)} .
$$

In summary,

$$
\left\|I[h]^{\prime} W\right\|_{L_{1}(c, d)} \leq\left(3\|\psi\|_{L_{\infty}(I)}\right)\|h W \psi\|_{L_{1}(c, d)} .
$$

Step 3: $1<p<\infty$.

We would like to interpolate this inequality to get an estimate of the norm of $\left(3\|\psi\|_{L_{\infty}(I)}\right)$ 
in any $L_{p}$ space. However, the standard interpolation theorems do not seem to apply to a space of functions restricted by the condition (45). Accordingly, we define (as did Mhaskar [24, p. 86 ff.])

$$
A_{0}[h]=\int_{I} h(\psi W)^{2} / \int_{I}(\psi W)^{2}
$$

and

$$
\mathcal{L}[h]=I\left[h-A_{0}[h]\right]^{\prime} .
$$

Note that $h-A_{0}[h]$ satisfies (45), that is,

$$
\int_{I}\left(h-A_{0}[h]\right)(\psi W)^{2}=0 .
$$

The above estimates show that, for $p=1$ and $p=\infty$,

$$
\begin{aligned}
\|\mathcal{L}[h] W\|_{L_{p}(c, d)} & \leq\left(3\|\psi\|_{L_{\infty}(I)}\right)\left\|\left(h-A_{0}[h]\right) W \psi\right\|_{L_{p}(c, d)} \\
& \leq\left(3\|\psi\|_{L_{\infty}(I)}\right)\left(\|h W \psi\|_{L_{p}(c, d)}+\left|A_{0}[h]\right|\|W \psi\|_{L_{p}(c, d)}\right) \\
& \leq\left(3\|\psi\|_{L_{\infty}(I)}\right)\|h W \psi\|_{L_{p}(c, d)}\left(1+\|W \psi\|_{L_{q}(c, d)} / \int_{I}(\psi W)^{2}\right),
\end{aligned}
$$

where $q$ is the dual parameter of $p$. The standard form of the Riesz-Thorin theorem gives, for $1<p<\infty$ and some $C$ depending on $p, W, \psi$,

$$
\|\mathcal{L}[h] W\|_{L_{p}(c, d)} \leq C_{p}\|h W \psi\|_{L_{p}(c, d)} .
$$

Then for functions $h$ satisfying (45), which is equivalent to $A_{0}[h]=0$, we have $\mathcal{L}[h]=$ $I[h]^{\prime}$ and so obtain (46).

We turn to

Proof of Theorem 1.5. Let $R$ be a polynomial satisfying (31) and (32). The Jackson inequality (33), followed by the lemma above gives

$$
\begin{aligned}
E_{n-2}[I[R] ; W] & \leq \eta_{n-2}\left\|I[R]^{\prime} W\right\|_{L_{2}(I)} \\
& \leq C_{2} \eta_{n-2}\|R W \psi\|_{L_{2}(I)}=C_{2} \eta_{n-2} .
\end{aligned}
$$

Thus (30) gives

$$
0 \leq\left(\frac{\gamma_{n-1}\left(W^{2}\right)}{n \kappa_{n}}\right)^{2}-\left\{\left(\frac{\gamma_{n-1}\left(W^{2}\right)}{n \gamma_{n}\left((\psi W)^{2}\right)}\right)^{2}+\lambda\right\} \leq C_{2}^{2} \eta_{n-2}^{2} .
$$

Here

$$
\begin{aligned}
\gamma_{n}^{-2}\left((\psi W)^{2}\right) & =\inf _{P \in \mathcal{P}_{n}^{m}} \int_{I} P^{2}(\psi W)^{2} \\
& \leq\|\psi\|_{L_{\infty}(I)}^{2} \inf _{P \in \mathcal{P}_{n}^{m}} \int_{I} P^{2} W^{2} \\
& =\|\psi\|_{L_{\infty}(I)}^{2} \gamma_{n}^{-2}\left(W^{2}\right) .
\end{aligned}
$$

Combining these last inequalities and rearranging gives (34). 
We record for future use

Lemma 2.3. Assume the hypotheses of Theorem 1.5. Then

$$
\begin{gathered}
\left\|\left(\frac{n}{\gamma_{n-1}\left(W^{2}\right)} \int_{0}^{x} p_{n-1}\left(W^{2}\right)-\frac{1}{\gamma_{n}\left((\psi W)^{2}\right)} p_{n}\left((\psi W)^{2}\right)\right) \psi W\right\|_{L_{2}(I)} \\
\leq C_{3} \frac{n}{\gamma_{n-1}\left(W^{2}\right)}\left[\eta_{n-2}+\left|\int_{I} p_{n-1}\left(W^{2}\right) \operatorname{sign}(\cdot) W^{2}\right|\right],
\end{gathered}
$$

where the constant $C_{3}$ is independent of $n$ but depends on $W, \psi$.

Proof. In view of Lemma 2.1, we must estimate the term $b_{0} p_{0}\left((\psi W)^{2}\right)$ that appears in the left-hand side of (44). We showed in the above proof that the right-hand side of (44) in Lemma 2.1 is bounded above by $C_{2} \eta_{n-2}\left(n / \gamma_{n-1}\left(W^{2}\right)\right)$. In estimating $b_{0}$, we can follow similar steps to that for $b_{j}, j \geq 1$, in the proof of Theorem 1.4, but extra difficulties arise because

$$
\int_{I} p_{0}\left((\psi W)^{2}\right)(\psi W)^{2} \neq 0
$$

Now as $p_{0}\left((\psi W)^{2}\right)$ is constant,

$$
\begin{aligned}
\frac{\gamma_{n-1}\left(W^{2}\right)}{n} b_{0} & =p_{0}\left((\psi W)^{2}\right)\left[\int_{c}^{0}+\int_{0}^{d}\right]\left[\int_{0}^{t} p_{n-1}\left(W^{2} ; x\right) d x\right](\psi W)^{2}(t) d t \\
& =p_{0}\left((\psi W)^{2}\right)\left(T_{1}+T_{2}\right),
\end{aligned}
$$

say. Integration by parts gives

$$
\begin{aligned}
& T_{1}=-\int_{c}^{0} p_{n-1}\left(W^{2} ; x\right)\left[\int_{c}^{x}(\psi W)^{2}(t) d t\right] d x, \\
& T_{2}=\int_{0}^{d} p_{n-1}\left(W^{2} ; x\right)\left[\int_{x}^{d}(\psi W)^{2}(t) d t\right] d x .
\end{aligned}
$$

(It is here that we have to proceed differently from $b_{j}, j \geq 1$, to circumvent (49).) Let

$$
f(x)=\operatorname{sign}(x) W^{-2}(x) \begin{cases}\int_{x}^{d}(\psi W)^{2}(t) d t, & x \in(0, d), \\ \int_{c}^{x}(\psi W)^{2}(t) d t, & x \in(c, 0] .\end{cases}
$$

Then

$$
\frac{\gamma_{n-1}\left(W^{2}\right)}{n p_{0}\left((\psi W)^{2}\right)} b_{0}=\int_{c}^{d} p_{n-1}\left(W^{2} ; x\right) f(x) W^{2}(x) d x .
$$

Observe that, since $W(0)=1$,

$$
f(0+)=\int_{0}^{d}(\psi W)^{2} \quad \text { and } \quad f(0-)=-\int_{c}^{0}(\psi W)^{2} .
$$


To take account of this jump discontinuity at 0 , we define a function $h$ such that $h(0 \pm)=$ $f(0 \pm)$, by

$$
h(x)=\left[\frac{1}{2} \int_{I}(\psi W)^{2}\right] \operatorname{sign}(x)+\frac{1}{2}\left[\int_{0}^{d}(\psi W)^{2}-\int_{c}^{0}(\psi W)^{2}\right] .
$$

Then $f-h$ has limit 0 at 0 , so is continuous there. Also, for a.e. $x>0$,

$$
(f-h)^{\prime}(x)=f^{\prime}(x)=2 Q^{\prime}(x) e^{2 Q(x)} \int_{x}^{d}(\psi W)^{2}-\psi^{2}(x)
$$

and, for a.e. $x<0$,

$$
(f-h)^{\prime}(x)=f^{\prime}(x)=-2 Q^{\prime}(x) e^{2 Q(x)} \int_{c}^{x}(\psi W)^{2}-\psi^{2}(x) .
$$

So $(f-h)^{\prime}+\psi^{2}$ has essential limit 0 at 0 , and then $f-h$ is absolutely continuous. Moreover, the convexity of $Q$ gives, as at (47),

$$
\left|(f-h)^{\prime}(x)\right| \leq 2\|\psi\|_{L_{\infty}(I)}^{2}, \quad \text { a.e. } x \in I .
$$

Next, split

$$
\begin{aligned}
\frac{\gamma_{n-1}\left(W^{2}\right)}{n p_{0}\left((\psi W)^{2}\right)} b_{0}= & \int_{c}^{d} p_{n-1}\left(W^{2} ; x\right)(f-h)(x) W^{2}(x) d x \\
& +\int_{c}^{d} p_{n-1}\left(W^{2} ; x\right) h(x) W^{2}(x) d x=\tau_{1}+\tau_{2} .
\end{aligned}
$$

Here

$$
\left|\tau_{1}\right| \leq E_{n-2}[f-h ; W] \leq \eta_{n-2}\left\|(f-h)^{\prime} W\right\|_{L_{2}(I)} \leq 2 \eta_{n-2}\|\psi\|_{L_{\infty}(I)}^{2}\|W\|_{L_{2}(I)},
$$

by our Jackson estimate (33) and our bound (50). Next, the definition of $h$ and orthogonality of $p_{n-1}$ to constants gives

$$
\left|\tau_{2}\right|=\left[\frac{1}{2} \int_{I}(\psi W)^{2}\right]\left|\int_{c}^{d} p_{n-1}\left(W^{2} ; x\right) \operatorname{sign}(x) W^{2}(x) d x\right| .
$$

Combining these last two relations, and that from Lemma 2.1 gives the result.

\section{A General Jackson Inequality}

We shall deduce Theorem 1.3 from Theorem 1.5 and the following crude, but general, Jackson inequality. More precise Jackson theorems for more restricted classes of weights can be found in [7], [8], [24]. In this section only, we use the notation

$$
E_{n, p}[f ; W]=\inf \left\{\|(f-P) W\|_{L_{p}(I)}: \operatorname{deg}(P) \leq n\right\} .
$$

In the notation of the Introduction, of course,

$$
E_{n}[f ; W]=E_{n, 2}[f ; W] .
$$


Theorem 3.1. Let $I=(c, d)$ be a finite or infinite interval containing 0 . Let $Q: I \rightarrow$ $[0, \infty)$ be convex and let $Q^{\prime}$ be absolutely continuous in $I$, with $Q^{\prime}(0)=0=Q(0)$ and $Q$ positive in $I \backslash\{0\}$. Assume also that, for $j=0,1$,

$$
\lim _{x \rightarrow c+}\left|Q^{(j)}(x)\right|=\infty=\lim _{x \rightarrow d-}\left|Q^{(j)}(x)\right| .
$$

Let $W=e^{-Q}$ and $1 \leq p<\infty$. Then there exists a decreasing sequence $\left(\eta_{n}\right)$ of positive numbers with limit 0 such that for every absolutely continuous function $f: I \rightarrow \mathbf{R}$ with $f^{\prime} W \in L_{p}(I)$, we have

$$
E_{n, p}[f ; W] \leq \eta_{n}\left\|f^{\prime} W\right\|_{L_{p}(I)} .
$$

We shall prove this in a series of lemmas. We may assume, by dilating $I$, that

$$
I \supset(-2,2) \text {. }
$$

Throughout this section, we use special notation. We shall use integers $n \geq 1$ and $m \ll n$, as well as parameters

$$
a_{-m} \leq \lambda_{-}<-1 \quad \text { and } \quad 1<\lambda_{+} \leq a_{m} .
$$

We denote by $\rho(m)$ an increasing function that depends on $m$ and $W$, while $\sigma\left(\lambda_{-}, \lambda_{+}\right)$ denotes a function increasing in $\lambda_{+}$and decreasing in $\lambda_{-}$. These functions change in different occurrences. The main feature is that $\sigma$ is independent of $m, n$, and functions $f$, while $\rho$ is independent of $\lambda_{ \pm}$, and functions $f$. At the end, we choose $m$ to grow slowly enough as a function of $n$, and then $\lambda_{ \pm}$also to approach the endpoints of $I$ sufficiently slowly.

Our strategy is to use the usual Jackson's theorem to approximate $f$ on $\left[\lambda_{-}, \lambda_{+}\right]$, and then to "damp down" this polynomial on $\left[a_{-m}, a_{m}\right] \backslash\left[\lambda_{-}, \lambda_{+}\right]$using fast-decreasing polynomials. Restricted range (or infinite-finite range) inequalities give the rest. We begin with a technical lemma.

Lemma 3.2. Assume the hypotheses on $Q$ in Theorem 3.1. Then:

(a) For $0<p \leq \infty$ and polynomials $P$ of degree $\leq n-2 / p$,

$$
\|P W\|_{L_{p}(I)} \leq 2^{1 / p}\|P W\|_{L_{p}\left[a_{-n}, a_{n}\right]} .
$$

(b)

$$
\lim _{n \rightarrow \infty} a_{-n}=c \quad \text { and } \quad \lim _{n \rightarrow \infty} a_{n}=d .
$$

(c)

$$
\lim _{n \rightarrow \infty} \delta_{n} / n=0 .
$$


Proof. (a) This is part of Theorem 4.1 in [15, p. 95].

(b) This follows from the convexity of $Q$. See, for example, Theorem 2.4 in [15, p. 41].

(c) If $I$ is finite, then $\left(\delta_{n}\right)$ is bounded and the result is immediate. Now assume that $I$ is unbounded. Let $n \geq 1$ and assume $a_{n} \geq\left|a_{-n}\right|$, so that $a_{n} \geq \delta_{n}$. Then, from (18), as $x Q^{\prime}(x) \geq 0$ in $I$, and as $Q^{\prime}$ is nondecreasing in $I$,

$$
\begin{aligned}
n & \geq \frac{1}{2} a_{n} Q^{\prime}\left(\frac{1}{2} a_{n}\right) \int_{(1 / 2) a_{n}}^{a_{n}} \frac{d x}{\sqrt{\left(x-a_{-n}\right)\left(a_{n}-x\right)}} \\
& \geq \frac{1}{2} \delta_{n} Q^{\prime}\left(\frac{1}{2} \delta_{n}\right) \int_{(1 / 2) a_{n}}^{a_{n}} \frac{d x}{\sqrt{\left(x+a_{n}\right)\left(a_{n}-x\right)}} \\
& =\frac{1}{2} \delta_{n} Q^{\prime}\left(\frac{1}{2} \delta_{n}\right) \int_{(1 / 2)}^{1} \frac{d t}{\sqrt{1-t^{2}}} .
\end{aligned}
$$

Similarly, if $a_{n}<\left|a_{-n}\right|$, we obtain

$$
n \geq \frac{1}{2} \delta_{n}\left|Q^{\prime}\left(-\frac{1}{2} \delta_{n}\right)\right| \int_{-1}^{-1 / 2} \frac{d t}{\sqrt{1-t^{2}}} .
$$

Since $\delta_{n} \rightarrow \infty$ as $n \rightarrow \infty$, and since $Q^{\prime}$ becomes unbounded at both endpoints of $I$, we then obtain the desired limit.

\section{Lemma 3.3.}

(a) There is a function $\sigma:(c, 0] \times[0, d) \rightarrow[0, \infty)$, decreasing in the first variable, and increasing in the second variable, with the following properties: let $m \geq 1$ and $\lambda_{ \pm}$satisfy (53). For all absolutely continuous $f$ with $f^{\prime} W \in L_{p}(I)$, there exists a polynomial $R_{m}$ of degree $\leq m$ such that

$$
\left\|\left(f-R_{m}\right) W\right\|_{L_{p}\left[\lambda_{-}, \lambda_{+}\right]} \leq \frac{\sigma\left(\lambda_{-}, \lambda_{+}\right)}{m}\left\|f^{\prime} W\right\|_{L_{p}(I)} .
$$

(b) Moreover, there is an increasing function $\rho: \mathbf{Z}_{+} \rightarrow(0, \infty)$ depending only on W such that

$$
\left\|R_{m} W\right\|_{L_{p}(I)}+\left\|R_{m} W\right\|_{L_{\infty}(I)} \leq \rho(m)\left(\|f W\|_{L_{p}(I)}+\left\|f^{\prime} W\right\|_{L_{p}(I)}\right) .
$$

Proof. (a) By the classical form of Jackson's theorem [6, Theorem 6.2, p. 219], translated from $[-1,1]$ to $\left[\lambda_{-}, \lambda_{+}\right]$, there exists $R_{m}$ with

$$
\left\|f-R_{m}\right\|_{L_{p}\left[\lambda_{-}, \lambda_{+}\right]} \leq C_{0} \frac{\lambda_{+}-\lambda_{-}}{m}\left\|f^{\prime}\right\|_{L_{p}\left[\lambda_{-}, \lambda_{+}\right]} .
$$

Here $C_{0}$ is an absolute constant, independent of $f, m, \lambda_{ \pm}$. Then

$$
\left\|\left(f-R_{m}\right) W\right\|_{L_{p}\left[\lambda_{-}, \lambda_{+}\right]} \leq C_{0} \frac{\lambda_{+}-\lambda_{-}}{m}\|W\|_{L_{\infty}\left[\lambda_{-}, \lambda_{+}\right]}\left\|\frac{1}{W}\right\|_{L_{\infty}\left[\lambda_{-}, \lambda_{+}\right]}\left\|f^{\prime} W\right\|_{L_{p}(I)} .
$$

So we can take

$$
\sigma\left(\lambda_{-}, \lambda_{+}\right)=C_{0}\left(\lambda_{+}-\lambda_{-}\right)\|W\|_{L_{\infty}\left[\lambda_{-}, \lambda_{+}\right]}\left\|\frac{1}{W}\right\|_{L_{\infty}\left[\lambda_{-}, \lambda_{+}\right]} .
$$


(b) By the restricted range inequalities in Lemma 3.2,

$$
\begin{gathered}
\left\|R_{m} W\right\|_{L_{p}(I)}+\left\|R_{m} W\right\|_{L_{\infty}(I)} \leq 2^{1 / p}\left\|R_{m} W\right\|_{L_{p}\left[a_{-m}, a_{m}\right]}+\left\|R_{m} W\right\|_{L_{\infty}\left[a_{-m}, a_{m}\right]} \\
\leq 2^{1 / p}\left\|R_{m}\right\|_{L_{\infty}\left[a_{-m}, a_{m}\right]}\left(\|W\|_{L_{p}(I)}+\|W\|_{L_{\infty}(I)}\right) .
\end{gathered}
$$

Recall the Chebyshev inequality [6, Proposition 2.3, p. 101], valid for polynomials $P$ of degree $\leq m$ :

$$
|P(x)| \leq\left|T_{m}(x)\right|\|P\|_{L_{\infty}[-1,1]}, \quad|x|>1 .
$$

Here $T_{m}$ is the classical Chebyshev polynomial of the first kind. By translating and dilating this, and using the bound

$$
\left|T_{m}(x)\right| \leq(2|x|)^{m}, \quad|x|>1,
$$

we obtain for some absolute constant $C_{0}$,

$$
\left\|R_{m}\right\|_{L_{\infty}\left[a_{-m}, a_{m}\right]} \leq\left(C_{0} \frac{a_{m}-a_{-m}}{\lambda_{+}-\lambda_{-}}\right)^{m}\left\|R_{m}\right\|_{L_{\infty}\left[\lambda_{-}, \lambda_{+}\right]} .
$$

Using the Nikolskii inequalities [6, Theorem 2.6, p. 102], we continue this for some absolute constant $C_{1}$ as

$$
\begin{aligned}
\left\|R_{m}\right\|_{L_{\infty}\left[a_{-m}, a_{m}\right]} & \leq\left(C_{0} \frac{a_{m}-a_{-m}}{\lambda_{+}-\lambda_{-}}\right)^{m}\left(\frac{C_{1} m^{2}}{\lambda_{+}-\lambda_{-}}\right)^{1 / p}\left\|R_{m}\right\|_{L_{p}\left[\lambda_{-}, \lambda_{+}\right]} \\
& \leq\left(C_{0} \delta_{m}\right)^{m}\left(C_{1} m\right)^{2 / p}\left\|R_{m}\right\|_{L_{p}\left[\lambda_{-}, \lambda_{+}\right]} \\
& \leq\left(C_{0} \delta_{m}\right)^{m}\left(C_{1} m\right)^{2 / p}\left(\|f\|_{L_{p}\left[\lambda_{-}, \lambda_{+}\right]}+C_{0} \frac{\lambda_{+}-\lambda_{-}}{m}\left\|f^{\prime}\right\|_{L_{p}\left[\lambda_{-}, \lambda_{+}\right]}\right),
\end{aligned}
$$

by the fact that $\left|\lambda_{ \pm}\right| \geq 1$ and by (56). Using our bound (53) on $\lambda_{ \pm}$, we continue this as

$$
\begin{aligned}
\left\|R_{m}\right\|_{L_{\infty}\left[a_{-m}, a_{m}\right]} \leq & \left(C_{0} \delta_{m}\right)^{m}\left(C_{1} m\right)^{2 / p}\left\|W^{-1}\right\|_{L_{\infty}\left[a_{-m}, a_{m}\right]} \\
& \times\left(1+C_{0} \frac{2 \delta_{m}}{m}\right)\left(\|f W\|_{L_{p}\left[\lambda_{-}, \lambda_{+}\right]}+\left\|f^{\prime} W\right\|_{L_{p}\left[\lambda_{-}, \lambda_{+}\right]}\right) .
\end{aligned}
$$

Combining this and (57) gives the result.

To handle the norm of $f W$ away from 0 , we use the following lemma. It is an extension of one that Mhaskar used extensively [24, pp. 75-76].

Lemma 3.4. Let $1 \leq p \leq \infty$, and let $W$ be as in Theorem 3.1. Assume in addition that

$$
\limsup _{x \rightarrow c+\text { or } x \rightarrow d-} \frac{Q^{\prime \prime}(x)}{Q^{\prime}(x)^{2}}<1 .
$$

(a) There exists $C>0$ such that, for $x \in I$,

$$
\left|Q^{\prime}(x) W(x) \int_{0}^{x} W^{-1}(t) d t\right| \leq C .
$$


(b) There exists $C_{p}$ depending only on $p, W$ such that

$$
\left\|Q^{\prime} g W\right\|_{L_{p}(I)} \leq C_{p}\left\|g^{\prime} W\right\|_{L_{p}(I)},
$$

for all $g: I \rightarrow \mathbf{R}$ absolutely continuous, with $g(0)=0$ and $g^{\prime} W \in L_{p}(I)$.

(c) Moreover under the same conditions on $g$, there exists $C_{p}$ depending only on $p$ and $W$ such that

$$
\left\|\left(\left|Q^{\prime}\right|+1\right) g W\right\|_{L_{p}(I)} \leq C_{p}\left\|g^{\prime} W\right\|_{L_{p}(I)} .
$$

Proof. (a) Choose $\varepsilon \in(0,1)$ and $A \in[0, d)$ such that

$$
x \in(A, d) \quad \Rightarrow \quad \frac{Q^{\prime \prime}(x)}{Q^{\prime}(x)^{2}}<1-\varepsilon .
$$

An integration by parts shows that

$$
\begin{aligned}
\int_{A}^{x} e^{Q(t)} d t & =\int_{A}^{x} Q^{\prime}(t)^{-1} \frac{d}{d t}\left(e^{Q(t)}\right) d t \\
& =Q^{\prime}(x)^{-1} e^{Q(x)}-Q^{\prime}(A)^{-1} e^{Q(A)}+\int_{A}^{x} \frac{Q^{\prime \prime}(t)}{Q^{\prime}(t)^{2}} e^{Q(t)} d t \\
& \leq Q^{\prime}(x)^{-1} e^{Q(x)}+(1-\varepsilon) \int_{A}^{x} e^{Q(t)} d t .
\end{aligned}
$$

So

$$
\int_{A}^{x} e^{Q(t)} d t \leq \varepsilon^{-1} Q^{\prime}(x)^{-1} e^{Q(x)} .
$$

Note that, for $x \in(A, d)$,

$$
\frac{d}{d x}\left[Q^{\prime}(x)^{-1} e^{Q(x)}\right]=e^{Q(x)}\left[1-\frac{Q^{\prime \prime}(x)}{Q^{\prime}(x)^{2}}\right]>0,
$$

so $Q^{\prime}(x)^{-1} e^{Q(x)}$ is increasing there. Then there exists $C>0$ such that, for $x \in[A, d)$,

$$
\int_{0}^{A} e^{Q(t)} d t \leq C Q^{\prime}(x)^{-1} e^{Q(x)} .
$$

So, for $x \in[A, d)$ and some $C_{1}$ independent of $x$,

$$
\int_{0}^{x} e^{Q(t)} d t \leq C_{1} Q^{\prime}(x)^{-1} e^{Q(x)} .
$$

Since the left-hand side is an increasing function of $x$, while $Q^{\prime}(x)^{-1}$ is a decreasing function of $x$, this inequality also holds for $x \in[0, A]$. So we have (59) for $x>0$. The case $x<0$ is similar.

(b) We do this first for $p=\infty$, then for $p=1$, and then interpolate, as did Mhaskar. 
Step 1: $p=\infty$.

Assume $x \in(0, d)$. Then, by (a),

$$
\begin{aligned}
\left|Q^{\prime} g W\right|(x) & =\left|Q^{\prime}(x) W(x) \int_{0}^{x}\left(g^{\prime} W\right)(t) W^{-1}(t) d t\right| \\
& \leq C\left\|g^{\prime} W\right\|_{L_{\infty}(I)} .
\end{aligned}
$$

Step 2: $p=1$.

Now as above

$$
\begin{aligned}
\int_{0}^{d}\left|Q^{\prime} g W\right|(x) d x & =\int_{0}^{d}\left|Q^{\prime}(x) W(x)\left[\int_{0}^{x}\left(g^{\prime} W\right)(t) W^{-1}(t) d t\right]\right| d x \\
& \leq \int_{0}^{d}\left|g^{\prime} W\right|(t)\left(\int_{t}^{d} Q^{\prime}(x) W(x) d x\right) W^{-1}(t) d t \\
& =\int_{0}^{d}\left|g^{\prime} W\right|(t) d t .
\end{aligned}
$$

A similar inequality holds in $(c, 0)$, so we obtain

$$
\left\|Q^{\prime} g W\right\|_{L_{1}(I)} \leq\left\|g^{\prime} W\right\|_{L_{1}(I)} .
$$

Step 3: $1<p<\infty$.

We have effectively shown that if we define the linear operator $\mathcal{L}$ on $L_{p}(I)$ by

$$
\mathcal{L}[h](x)=Q^{\prime}(x) W(x) \int_{0}^{x} h(t) W^{-1}(t) d t
$$

(above $h=g^{\prime} W$ ), then, for $p=1$ and $p=\infty$,

$$
\|\mathcal{L}[h]\|_{L_{p}(I)} \leq C_{p}\|h\|_{L_{p}(I)} .
$$

Now the Riesz-Thorin theorem [2, Theorem 3.6, p. 213] shows that this holds in $L_{p}(I)$ for any $1<p<\infty$. Taking $h=g^{\prime} W$ then gives (60).

(c) Because $Q^{\prime}$ becomes unbounded at $c$ or $d$, while $W^{ \pm 1}$ are bounded in any compact subinterval of $I$, it suffices to show that for finite $c<r<0<s<d$, there exists $C>0$ (depending on $r, s$, and $p$ ) such that

$$
\|g\|_{L_{p}[r, s]} \leq C_{p}\left\|g^{\prime}\right\|_{L_{p}[r, s]} .
$$

Since

$$
\begin{aligned}
\|g\|_{L_{p}[r, s]} & =\left(\int_{r}^{s}\left|\int_{0}^{x} g^{\prime}(t) d t\right|^{p} d x\right)^{1 / p} \\
& \leq\left(\int_{r}^{s}\left\|g^{\prime}\right\|_{L_{p}[r, s]}^{p}|x|^{p-1} d x\right)^{1 / p},
\end{aligned}
$$

the desired inequality follows. 
Since we do not assume (58) in Theorem 3.1, we also need a weaker version of Lemma 3.4 that avoids that hypothesis.

Lemma 3.5. Assume $W$ is as in Theorem 3.1. There exists a positive increasing function $\Phi: I \rightarrow[0, \infty)$ such that

$$
\lim _{x \rightarrow c+\text { or } x \rightarrow d_{-}}|\Phi(x)|=\infty
$$

and with the following properties: Let $g: I \rightarrow \mathbf{R}$ be absolutely continuous, with $g(0)=0$ and $g^{\prime} W \in L_{p}(I)$.

(a) There exists $C>0$ such that, for $x \in I$,

$$
|\Phi(x)| e^{-Q(x)} \int_{0}^{x} e^{Q(t)} d t \leq C .
$$

(b) There exists $C_{p}$ depending only on $p$ and $W$ such that

$$
\|(|\Phi|+1) g W\|_{L_{p}(I)} \leq C_{p}\left\|g^{\prime} W\right\|_{L_{p}(I)} .
$$

Proof. (a) Let $x>0$ be such that

$$
Q(x)>2 Q(1)
$$

and choose $A=A(x)>1$ to be the smallest number such that

$$
Q(A)=\frac{1}{2} Q(x)
$$

(This will be possible as $Q$ is continuous and increasing.) Since $Q^{\prime}$ is increasing,

$$
\begin{aligned}
\int_{A}^{x} e^{Q(t)} d t & \leq \int_{A}^{x} \frac{Q^{\prime}(t)}{Q^{\prime}(A)} e^{Q(t)} d t \\
& =Q^{\prime}(A)^{-1}\left(e^{Q(x)}-e^{Q(A)}\right) \leq Q^{\prime}(A)^{-1} e^{Q(x)}
\end{aligned}
$$

Also,

$$
\int_{1}^{A} e^{Q(t)} d t \leq Q^{\prime}(1)^{-1} e^{Q(A)}=Q^{\prime}(1) e^{(1 / 2) Q(x)}
$$

Then

$$
e^{-Q(x)} \int_{0}^{x} e^{Q(t)} d t \leq e^{-Q(x)} \int_{0}^{1} e^{Q(t)} d t+Q^{\prime}(1) e^{-(1 / 2) Q(x)}+Q^{\prime}(A)^{-1} .
$$

Let $Q_{+}^{[-1]}$ denote the lower inverse function of $Q$ when restricted to $[0, d)$, so that $Q_{+}^{[-1]}:[0, \infty) \rightarrow[0, d)$ and

$$
Q_{+}^{[-1]}(y)=\min \{x \in[0, d): Q(x)=y\} .
$$

Then

$$
A=Q_{+}^{[-1]}\left(\frac{1}{2} Q(x)\right)
$$


Let

$$
\Phi(x)^{-1}=e^{-Q(x)} \int_{0}^{1} e^{Q(t)} d t+Q^{\prime}(1) e^{-(1 / 2) Q(x)}+Q^{\prime}\left(Q_{+}^{[-1]}\left(\frac{1}{2} Q(x)\right)\right)^{-1} .
$$

Then we have, by (66),

$$
\Phi(x) e^{-Q(x)} \int_{0}^{x} e^{Q(t)} d t \leq 1
$$

Also

$$
Q_{+}^{[-1]}\left(\frac{1}{2} Q(x)\right) \leq Q_{+}^{[-1]}(Q(x)) \leq x,
$$

so

$$
\Phi(x)^{-1} \geq Q^{\prime}\left(Q_{+}^{[-1]}\left(\frac{1}{2} Q(x)\right)\right)^{-1} \geq Q^{\prime}(x)^{-1} .
$$

Moreover, we see that $\Phi$ is increasing to $\infty$ as $x \rightarrow d$ with limit $\infty$ there. For $x>0$ for which (65) fails, we use the fact that $e^{-Q(x)} \int_{0}^{x} e^{Q(t)} d t$ is bounded in a compact subinterval of $[0, d)$, and can just define $\Phi$ to be constant there. Similarly, we handle $x<0$.

(b) One proceeds much as in Lemma 3.4. For $p=\infty$, the inequality (63) ensures (as in Lemma 3.4) that

$$
\|(|\Phi|+1) g W\|_{L_{\infty}(I)} \leq C_{p}\left\|g^{\prime} W\right\|_{L_{\infty}(I)} .
$$

Next, note that for $p=1$ in Lemma 3.4, we did not use (58). Using (67) and its analogues for other ranges of $x$, we have

$$
|\Phi| \leq C\left(\left|Q^{\prime}\right|+1\right)
$$

and then the case $p=1$ of Lemma 3.4(b), (c) give

$$
\|(|\Phi|+1) g W\|_{L_{1}(I)} \leq C_{p}\left\|g^{\prime} W\right\|_{L_{1}(I)} .
$$

Now interpolation for $1<p<\infty$ gives the rest.

Next, we need fast decreasing polynomials: $\chi_{S}$ denotes the characteristic function of a set $S$.

Lemma 3.6. Let

$$
-1 \leq r<s \leq 1
$$

There exists $C>0$, and for $n \geq 1$, polynomials $U_{n}$ of degree $\leq n / 2$ such that, for $x \in[-1,1]$,

$$
\left|U_{n}(x)-\chi_{[r, s]}(x)\right| \leq C \exp \left(-\left[\frac{n}{8} \min \{|x-r|,|x-s|\}\right]^{1 / 2}\right) .
$$

Here $C$ is independent of $n, x, r, s$. 
Proof. Nevai and Totik [30, Corollary 2, p. 117] showed that there exist polynomials $P_{n}$ of degree $\leq n / 4$ such that, for $x \in[-1,1]$,

$$
\left|P_{n}(x)-\operatorname{sign}\right|(x) \leq C \exp \left(-\left[\frac{n}{4}|x|\right]^{1 / 2}\right) .
$$

The constant $C$ is independent of $n$ and $x$. For $a \in[0,1]$, we set

$$
S_{n, a}(x)=\frac{1}{2}\left(1+P_{n}\left(\frac{x-a}{1+a}\right)\right) .
$$

Since $x \in[-1,1] \Rightarrow(x-a) /(1+a) \in[-1,1]$, and since (except at $x=a)$,

$$
\chi_{(a, \infty)}(x)=\frac{1}{2}\left(1+\operatorname{sign}\left(\frac{x-a}{1+a}\right)\right)
$$

we have

$$
\begin{aligned}
\left|S_{n, a}(x)-\chi_{(a, \infty)}(x)\right| & \leq C \exp \left(-\left[\frac{n}{4}\left|\frac{x-a}{1+a}\right|\right]^{1 / 2}\right) \\
& \leq C \exp \left(-\left[\frac{n}{8}|x-a|\right]^{1 / 2}\right) .
\end{aligned}
$$

For $a \in[-1,0)$, we set

$$
S_{n, a}(x)=1-S_{n,-a}(-x),
$$

and see that it admits a similar estimate. Now we set

$$
U_{n}(x)=S_{n, r}(x)\left(1-S_{n, s}(x)\right)
$$

and use

$$
\chi_{[r, s]}(x)=\chi_{(r, \infty)}(x)\left(1-\chi_{(s, \infty)}(x)\right)
$$

(except at $x=r$ ) to deduce that

$$
\left|U_{n}(x)-\chi_{[r, s]}(x)\right| \leq C \exp \left(-\left[\frac{n}{8}|x-r|\right]^{1 / 2}+\exp \left(-\left[\frac{n}{8}|x-s|\right]^{1 / 2}\right)\right) .
$$

From this, we conclude:

Lemma 3.7. With the restrictions on $\lambda_{ \pm}$in (53), there exist polynomials $V_{n}$ of degree $\leq n / 2$ such that, for $x \in\left[a_{-2 n}-1, a_{2 n}+1\right]$,

$$
\left|\chi_{\left[\lambda_{-}, \lambda_{+}\right]}(x)-V_{n}(x)\right| \leq C \exp \left(-\left[\frac{n}{8\left(\delta_{2 n}+1\right)} \min \left\{\left|x-\lambda_{-}\right|,\left|x-\lambda_{+}\right|\right\}\right]^{1 / 2}\right) .
$$

Here $C$ is independent of $n$ and $x$. 
Proof. Let $\ell_{n}$ denote the linear map of $\left[a_{-2 n}-1, a_{2 n}+1\right]$ onto $[-1,1]$, so that

$$
\ell_{n}(x)=-1+\frac{x-\left(a_{-2 n}-1\right)}{\delta_{2 n}+1} .
$$

Let

$$
r=\ell_{n}\left(\lambda_{-}\right) \quad \text { and } \quad s=\ell_{n}\left(\lambda_{+}\right),
$$

and

$$
V_{n}(x)=U_{n}\left(\ell_{n}(x)\right) .
$$

Then (except possibly at $\left.\lambda_{-}, \lambda_{+}\right)$,

$$
\begin{aligned}
\left|\chi_{\left[\lambda_{-}, \lambda_{+}\right]}(x)-V_{n}(x)\right| & =\left|\chi_{[r, s]}\left(\ell_{n}(x)\right)-U_{n}\left(\ell_{n}(x)\right)\right| \\
& \leq C \exp \left(-\left[\frac{n}{8} \min \left\{\left|\ell_{n}(x)-\ell_{n}\left(\lambda_{-}\right)\right|,\left|\ell_{n}(x)-\ell_{n}\left(\lambda_{+}\right)\right|\right\}\right]^{1 / 2}\right) \\
& \leq C \exp \left(-\left[\frac{n}{8\left(\delta_{2 n}+1\right)} \min \left\{\left|x-\lambda_{-}\right|,\left|x-\lambda_{+}\right|\right\}\right]^{1 / 2}\right) .
\end{aligned}
$$

Finally, we can give

Proof of Theorem 3.1. We may assume that $f(0)=0$. (If not, replace $f$ by $f-f(0)$ and absorb the constant $f(0)$ into the approximating polynomial.) We choose $n \geq 1$ and $1 \leq m \leq n / 2$, and let $\lambda_{ \pm}$satisfy (53). Let $R_{m}$ and $V_{n}$ denote the polynomials of Lemma 3.3 and 3.7, respectively, and let

$$
P_{n}=R_{m} V_{n}
$$

Then $P_{n}$ is a polynomial of degree $\leq n$, and

$$
\begin{aligned}
E_{n, p}[f ; W] \leq & \left\|\left(f-P_{n}\right) W\right\|_{L_{p}(I)} \\
\leq & \left\|\left(f-P_{n}\right) W\right\|_{L_{p}\left[\lambda_{-}, \lambda_{+}\right]}+\|f W\|_{L_{p}\left(I \backslash\left[\lambda_{-}, \lambda_{+}\right]\right)} \\
& +\left\|P_{n} W\right\|_{\left.L_{p}\left(I \backslash \lambda_{-}, \lambda_{+}\right]\right)} \\
= & T_{1}+T_{2}+T_{3} .
\end{aligned}
$$

First,

$$
\begin{aligned}
T_{1} \leq & \left\|\left(f-R_{m}\right) W\right\|_{L_{p}\left[\lambda_{-}, \lambda_{+}\right]}+\left\|R_{m}\left(1-V_{n}\right) W\right\|_{L_{p}\left[\lambda_{-}, \lambda_{+}\right]} \\
\leq & \left\|\left(f-R_{m}\right) W\right\|_{L_{p}\left[\lambda_{-}, \lambda_{+}\right]}+\left\|R_{m} W\right\|_{L_{\infty}\left[\lambda_{-}, \lambda_{+}\right]}\left\|1-V_{n}\right\|_{L_{p}\left[\lambda_{-}, \lambda_{+}\right]} \\
\leq & \frac{\sigma\left(\lambda_{-}, \lambda_{+}\right)}{m}\left\|f^{\prime} W\right\|_{L_{p}(I)} \\
& +\rho(m)\left(\|f W\|_{L_{p}(I)}+\left\|f^{\prime} W\right\|_{L_{p}(I)}\right)\left\|1-V_{n}\right\|_{L_{p}\left[\lambda_{-}, \lambda_{+}\right]},
\end{aligned}
$$

by Lemma 3.3(a), (b). Here by Lemma 3.5(b), for some $C$ independent of $f, m, n, \lambda_{ \pm}$,

$$
\|f W\|_{L_{p}(I)} \leq C\left\|f^{\prime} W\right\|_{L_{p}(I)} .
$$


Moreover, by Lemma 3.7,

$$
\begin{aligned}
\left\|1-V_{n}\right\|_{L_{p}\left[\lambda_{-}, \lambda_{+}\right]}^{p} & \leq C \int_{\lambda_{-}}^{\lambda_{+}} \exp \left(-p\left[\frac{n}{8\left(\delta_{2 n}+1\right)} \min \left\{\left|x-\lambda_{-}\right|,\left|x-\lambda_{+}\right|\right\}\right]^{1 / 2}\right) d x \\
& \leq C\left(\frac{\delta_{2 n}+1}{n}\right)
\end{aligned}
$$

with $C$ independent of $f, m, n, \lambda_{ \pm}$. Combining all these gives

$$
T_{1} \leq\left\|f^{\prime} W\right\|_{L_{p}(I)}\left\{\frac{\sigma\left(\lambda_{-}, \lambda_{+}\right)}{m}+\rho(m)\left(\frac{\delta_{2 n}+1}{n}\right)^{1 / p}\right\} .
$$

The crucial thing here is that $\sigma$ and $\rho$ are independent of $f, n$. Next, if $\Phi$ is as in Lemma 3.5,

$$
\begin{aligned}
T_{2} & \leq\|\Phi f W\|_{L_{p}\left(I \backslash\left[\lambda_{-}, \lambda_{+}\right]\right)}\left\|\frac{1}{\Phi}\right\|_{L_{\infty}\left(I \backslash\left[\lambda_{-}, \lambda_{+}\right]\right)} \\
& \leq\left\|f^{\prime} W\right\|_{L_{p}\left(I \backslash\left[\lambda_{-}, \lambda_{+}\right]\right)}\left\|\frac{1}{\Phi}\right\|_{L_{\infty}\left(I \backslash\left[\lambda_{-}, \lambda_{+}\right]\right)}
\end{aligned}
$$

by Lemma 3.5(b). Finally,

$$
\begin{aligned}
T_{3} \leq & \left\|P_{n} W\right\|_{L_{p}\left(\left[a_{-2 n}-1, a_{2 n}+1\right] \cap I \backslash\left[\lambda_{-}, \lambda_{+}\right]\right)}+\left\|P_{n} W\right\|_{L_{p}\left(I \backslash\left[a_{-2 n}-1, a_{2 n}+1\right]\right)} \\
& =: T_{31}+T_{32}
\end{aligned}
$$

Here

$$
T_{31} \leq\left\|R_{m} W\right\|_{L_{\infty}(I)}\left\|V_{n}\right\|_{L_{p}\left(\left[a_{-2 n}-1, a_{2 n}+1\right] \cap I \backslash\left[\lambda_{-}, \lambda_{+}\right]\right)} .
$$

For the first term in the product on the right-hand side, we can use Lemma 3.3(b) and (70). For the second term, we can use Lemma 3.7:

$$
\begin{aligned}
& \left\|V_{n}\right\|_{L_{p}\left(\left[a_{-2 n}-1, a_{2 n}+1\right] \cap I \backslash\left[\lambda_{-}, \lambda_{+}\right]\right)}^{p} \exp \left(-p\left[\frac{n}{8\left(\delta_{2 n}+1\right)} \min \left\{\left|x-\lambda_{-}\right|,\left|x-\lambda_{+}\right|\right\}\right]^{1 / 2}\right) d x \\
& \quad \leq C \int_{I \backslash\left[\lambda_{-}, \lambda_{+}\right]} \\
& \quad \leq C_{1} \frac{\delta_{2 n}+1}{n} .
\end{aligned}
$$

Thus

$$
T_{31} \leq \rho(m)\left\|f^{\prime} W\right\|_{L_{p}(I)}\left(\frac{\delta_{2 n}+1}{n}\right)^{1 / p} .
$$

Next, let $\ell_{n}$ denote the linear map of $\left[a_{-2 n}, a_{2 n}\right]$ onto $[-1,1]$, so that

$$
\ell_{n}(x)=-1+\frac{x-a_{-2 n}}{\delta_{2 n}}
$$


We see that, for $x \geq a_{2 n}+1$,

$$
\ell_{n}(x) \geq \ell_{n}\left(a_{2 n}\right)+\frac{1}{\delta_{2 n}}=1+\frac{1}{\delta_{2 n}},
$$

so

$$
\ell_{n}^{n}(x) \geq\left(1+\frac{1}{\delta_{2 n}}\right)^{n} \geq \exp \left(C \frac{n}{\delta_{2 n}}\right) .
$$

A similar estimate holds for $x \leq a_{-2 n}-1$. Then

$$
\begin{aligned}
T_{32} & \leq \exp \left(-C \frac{n}{\delta_{2 n}}\right)\left\|\ell_{n}^{n} P_{n} W\right\|_{L_{p}\left(I \backslash\left[a_{-2 n}-1, a_{2 n}+1\right]\right)} \\
& \leq 2^{1 / p} \exp \left(-C \frac{n}{\delta_{2 n}}\right)\left\|\ell_{n}^{n} P_{n} W\right\|_{L_{p}\left[a_{-2 n}, a_{2 n}\right]},
\end{aligned}
$$

by the restricted range inequality in Lemma 3.2(a). Then as $\left|\ell_{n}\right| \leq 1$ in $\left[a_{-2 n}, a_{2 n}\right]$, while $V_{n}$ is bounded (independent of $n$ there), we obtain

$$
\begin{aligned}
T_{32} & \leq C \exp \left(-C \frac{n}{\delta_{2 n}}\right)\left\|R_{m} W\right\|_{L_{p}\left[a_{-2 n}, a_{2 n}\right]} \\
& \leq \exp \left(-C \frac{n}{\delta_{2 n}}\right) \rho(m)\left\|f^{\prime} W\right\|_{L_{p}(I)} .
\end{aligned}
$$

Combining all the estimates (namely, (68), (71), (72), (73)) gives

$$
E_{n, p}[f ; W] \leq\left\|f^{\prime} W\right\|_{L_{p}(I)}\left\{\begin{array}{c}
\frac{\sigma\left(\lambda_{-}, \lambda_{+}\right)}{m}+\left\|\frac{1}{\Phi}\right\|_{L_{\infty}\left(I \backslash\left[\lambda_{-}, \lambda_{+}\right]\right)} \\
+\rho(m)\left[\left(\frac{\delta_{2 n}+1}{n}\right)^{1 / p}+\exp \left(-C \frac{n}{\delta_{2 n}}\right)\right]
\end{array}\right\} .
$$

The functions $\sigma$ and $\rho$ obey the conventions listed at the beginning of this section, and are independent of $f$. For a given large enough $n \geq 1$, we choose $m=m(n)$ to be the largest integer $\leq n / 2$ such that

$$
\rho(m)\left[\left(\frac{\delta_{2 n}+1}{n}\right)^{1 / p}+\exp \left(-C \frac{n}{\delta_{2 n}}\right)\right] \leq\left(\frac{\delta_{2 n}}{n}\right)^{1 /(2 p)} .
$$

Since (by Lemma 3.2(c)) $\delta_{2 n} / n \rightarrow 0$ as $n \rightarrow \infty$, necessarily $m=m(n)$ approaches $\infty$ as $n \rightarrow \infty$. Next, for the given $m=m(n)$, we choose the largest $t \leq m$ such that

$$
\sigma\left(a_{-t}, a_{t}\right) \leq \sqrt{m}
$$

and then set $\lambda_{-}=a_{-t}$ and $\lambda_{+}=a_{t}$. Then (at least for large $n$ and $m=m(n)$ ), (53) will be satisfied, and

$$
\frac{\sigma\left(\lambda_{-}, \lambda_{+}\right)}{m} \leq \frac{1}{\sqrt{m}}
$$


As $\sigma$ is finite valued, necessarily $\lambda_{+} \rightarrow d$ and $\lambda_{-} \rightarrow c$ as $n \rightarrow \infty$, so (recall (62))

$$
\left\|\frac{1}{\Phi}\right\|_{L_{\infty}\left(I \backslash\left[\lambda_{-}, \lambda_{+}\right]\right)} \rightarrow 0, \quad n \rightarrow \infty .
$$

Then denoting the term in \{\} in (74) by $\eta_{n}$, we have that $\left(\eta_{n}\right)$ has limit 0 as $n \rightarrow \infty$, and is independent of $f$. By a straightforward argument, we may modify them so that they are decreasing.

\section{Proof of Theorem 1.3}

Proof of (21) of Theorem 1.3. First, recall the estimate (34) of Theorem 1.5:

$$
\left|\left(\frac{\gamma_{n-1}\left(W^{2}\right)}{n \kappa_{n}}\right)^{2}-\lambda\right| \leq\left(\|\psi\|_{L_{\infty}(I)} \frac{\gamma_{n-1}\left(W^{2}\right)}{n \gamma_{n}\left(W^{2}\right)}\right)^{2}+C_{1} \eta_{n-2}^{2} .
$$

We shall show that

$$
\frac{\gamma_{n-1}\left(W^{2}\right)}{\gamma_{n}\left(W^{2}\right)} \leq 4 \delta_{n}
$$

and then using Theorem 3.1, and Lemma 3.2(c),

$$
\left|\left(\frac{\gamma_{n-1}\left(W^{2}\right)}{n \kappa_{n}}\right)^{2}-\lambda\right|=O\left(\frac{\delta_{n}}{n}\right)^{2}+O\left(\eta_{n}^{2}\right)=o(1) .
$$

To prove (75), we use the well-known identity

$$
\frac{\gamma_{n-1}\left(W^{2}\right)}{\gamma_{n}\left(W^{2}\right)}=\int_{I} x p_{n-1}(x) p_{n}(x) W^{2}(x) d x .
$$

If we apply the restricted range inequality in Lemma 3.2(a), applied with $p=1$ and to $W^{2}$ rather than $W$, and if we use the fact that $a_{ \pm 2 n}$ for $W^{2}$ is $a_{ \pm n}$ for $W$, we obtain

$$
\begin{aligned}
\frac{\gamma_{n-1}\left(W^{2}\right)}{\gamma_{n}\left(W^{2}\right)} & \leq 2 \int_{a_{-n}}^{a_{n}}\left|x p_{n-1}(x) p_{n}(x) W^{2}(x)\right| d x \\
& \leq 2 \max \left\{a_{n},\left|a_{-n}\right|\right\} \int_{a_{-n}}^{a_{n}}\left|p_{n-1}(x) p_{n}(x) W^{2}(x)\right| d x \\
& \leq 4 \delta_{n},
\end{aligned}
$$

as desired.

Next, define a polynomial $\pi_{n}(x)$ of degree $\leq n-2$ by

$$
\pi_{n}(x)=q_{n}^{\prime}-n \frac{\kappa_{n}}{\gamma_{n-1}\left(W^{2}\right)} p_{n-1} .
$$


We see that by orthogonality of $p_{n-1} W^{2}$ to polynomials of degree $\leq n-2$,

$$
\begin{aligned}
\int_{I} \pi_{n}^{2} W^{2} & =\int_{I}\left(q_{n}^{\prime}-n \frac{\kappa_{n}}{\gamma_{n-1}\left(W^{2}\right)} p_{n-1}\right)(x) \pi_{n}(x) W^{2}(x) d x \\
& =\int_{I} q_{n}^{\prime}(x) \pi_{n}(x) W^{2}(x) d x \\
& =\int_{I}\left(q_{n}^{\prime}\right)^{2} W^{2}-n \frac{\kappa_{n}}{\gamma_{n-1}\left(W^{2}\right)} \int_{I} q_{n}^{\prime} p_{n-1} W^{2} \\
& =\frac{1}{\lambda}\left[1-\int_{I} q_{n}^{2}(\psi W)^{2}\right]-\left(n \frac{\kappa_{n}}{\gamma_{n-1}\left(W^{2}\right)}\right)^{2} .
\end{aligned}
$$

Here we have used the definition of the inner product, the normalization $\left(q_{n}, q_{n}\right)=1$, and orthogonality. The identity (77) also implies, as its left-hand side is nonnegative,

$$
\int_{I} q_{n}^{2}(\psi W)^{2} \leq 1-\lambda\left(n \frac{\kappa_{n}}{\gamma_{n-1}\left(W^{2}\right)}\right)^{2} .
$$

Now we continue (77) as

$$
\begin{aligned}
\int_{I}\left(q_{n}^{\prime}-n \frac{\kappa_{n}}{\gamma_{n-1}\left(W^{2}\right)} p_{n-1}\right)^{2} W^{2} & \leq \frac{1}{\lambda}-\left(n \frac{\kappa_{n}}{\gamma_{n-1}\left(W^{2}\right)}\right)^{2} \\
& =o(1),
\end{aligned}
$$

by (76). Then orthogonality of $p_{n-1}$ to polynomials of degree $\leq n-2$ gives

$$
\begin{aligned}
\int_{I}\left(q_{n}^{\prime}-\frac{1}{\sqrt{\lambda}}\right. & \left.p_{n-1}\right)^{2} W^{2} \\
= & \int_{I}\left(q_{n}^{\prime}-n \frac{\kappa_{n}}{\gamma_{n-1}\left(W^{2}\right)} p_{n-1}\right)^{2} W^{2} \\
& +\int_{I}\left(n \frac{\kappa_{n}}{\gamma_{n-1}\left(W^{2}\right)}-\frac{1}{\sqrt{\lambda}}\right)^{2} p_{n-1}^{2} W^{2}=o(1) .
\end{aligned}
$$

Proof of (23) of Theorem 1.3 assuming (22). Since

$$
\left[q_{n}(x)-q_{n}(0)-\frac{1}{\sqrt{\lambda}} \int_{0}^{x} p_{n-1}\right]_{\mid x=0}=0
$$

Lemma 3.4(c) gives

$$
\begin{gathered}
\left\|\left(\left|Q^{\prime}\right|+1\right)\left[q_{n}-q_{n}(0)-\frac{1}{\sqrt{\lambda}} \int_{0}^{x} p_{n-1}\right] W\right\|_{L_{2}(I)} \\
\leq C\left\|\left[q_{n}^{\prime}-\frac{1}{\sqrt{\lambda}} p_{n-1}\right] W\right\|_{L_{2}(I)}=o(1) .
\end{gathered}
$$

For the asymptotics in the plane, we need an estimate for polynomials in the plane, in terms of their values on a segment. 
Lemma 4.1. Let $1 \leq p \leq \infty$ and let $w \in L_{p}[-1,1]$ be a nonnegative function. Let

$$
\widetilde{w}(\theta)=|\sin \theta|^{1 / p} w(\cos \theta), \quad \theta \in[-\pi, \pi],
$$

satisfy Szegö's condition (4). Let $P$ be a polynomial of degree $\leq m$. Then, for $z \in$ $\mathbf{C} \backslash[-1,1]$,

$$
\left|\frac{P(z)}{\varphi(z)^{m}}\right| \leq\|P w\|_{L_{p}[-1,1]} \pi^{-1 / p}\left|\frac{D^{-2}(\widetilde{w} ; 1 / \varphi(z))}{1-|\varphi(z)|^{-1}}\right| .
$$

Proof. See, for example, Lemma 14.6 in [15, p. 395].

Proof of (25). Let $L_{n}$ denote the linear map of $\left[a_{-n}, a_{n}\right]$ onto $[-1,1]$, let $L_{n}^{[-1]}$ denote its inverse, and let, as at (24),

$$
W_{n}(\theta)=W\left(L_{n}^{[-1]}(\cos \theta)\right), \quad \theta \in[-\pi, \pi],
$$

and

$$
\widetilde{W}_{n}(\theta)=W_{n}(\theta)|\sin \theta|^{1 / 2}, \quad \theta \in[-\pi, \pi] .
$$

Then the lemma gives (with $p=2, m=n-1, w=W_{n}$ )

$$
\begin{aligned}
& \left|\frac{\left(q_{n}^{\prime}-(1 / \sqrt{\lambda}) p_{n-1}\right)\left(L_{n}^{[-1]}(z)\right)}{\varphi(z)^{n-1} D^{-2}\left(\widetilde{W}_{n} ; 1 / \varphi(z)\right)}\right|\left(1-|\varphi(z)|^{-1}\right) \\
& \quad \leq \pi^{-1 / 2}\left\|\left[\left(q_{n}^{\prime}-\frac{1}{\sqrt{\lambda}} p_{n-1}\right) \circ L_{n}^{[-1]}\right] W \circ L_{n}^{[-1]}\right\|_{L_{2}[-1,1]} \\
& \quad=\pi^{-1 / 2} \delta_{n}^{-1 / 2}\left\|\left(q_{n}^{\prime}-\frac{1}{\sqrt{\lambda}} p_{n-1}\right) W\right\|_{L_{2}\left[a_{-n}, a_{n}\right]}=o\left(\delta_{n}^{-1 / 2}\right) .
\end{aligned}
$$

In each closed subset of $\mathbf{C} \backslash[-1,1]$, there exists $r>1$ such that in that set

$$
|\varphi| \geq r>1 \text {. }
$$

Moreover,

$$
\left|D^{-2}\left(\widetilde{W}_{n} ; \frac{1}{\varphi(z)}\right) / D^{-2}\left(W_{n} ; \frac{1}{\varphi(z)}\right)\right|=\left|D^{-2}\left(|\sin \cdot|^{1 / 2} ; \frac{1}{\varphi(z)}\right)\right|
$$

is bounded above and below in such a set. Then (25) follows.

Proof of (27). This is very similar to that above, just apply Lemma 4.1 to

$$
q_{n}\left(L_{n}(z)\right)-q_{n}(0)-\frac{1}{\sqrt{\lambda}} \int_{0}^{L_{n}(z)} p_{n-1}
$$

and with $W_{n}^{*}$ replacing $W_{n}$. We obtain

$$
\begin{aligned}
& \left|\frac{q_{n}\left(L_{n}(z)\right)-q_{n}(0)-(1 / \sqrt{\lambda}) \int_{0}^{L_{n}(z)} p_{n-1}}{\varphi(z)^{n} D^{-2}\left(W_{n}^{*} ; 1 / \varphi(z)\right)}\right|\left(1-|\varphi(z)|^{-1}\right) \\
& \leq \pi^{-1 / 2} \delta_{n}^{-1 / 2}\left\|\left(1+\left|Q^{\prime}\right|\right)\left(q_{n}-q_{n}(0)-\frac{1}{\sqrt{\lambda}} \int_{0}^{x} p_{n-1}\right) W\right\|_{L_{2}\left[a_{-n}, a_{n}\right]} \\
& \quad=o(1) .
\end{aligned}
$$




\section{Proof of Theorem 1.1 and Corollary 1.2}

We begin with a technical lemma:

Lemma 5.1. Assume that $Q$ is as in Theorem 1.1:

(a)

$$
\lim _{x \rightarrow \infty} \frac{Q^{\prime \prime}(x)}{Q^{\prime}(x)^{2}}=0 .
$$

(b) For $n \geq 1$ and polynomials $P$ of degree $\leq n$,

$$
\|P W\|_{L_{\infty}(\mathbf{R})} \leq C\left(\frac{n}{a_{n}}\right)^{1 / 2}\|P W\|_{L_{2}(\mathbf{R})} .
$$

(c) Let $n \geq 1$ and let

$$
g_{n}(\theta)=1+\left|Q^{\prime}\left(a_{n} \cos \theta\right)\right|, \quad \theta \in[-\pi, \pi] .
$$

Let $0<r<1$. There exists $C>1$ such that, for $|z| \leq r$ and $n \geq 1$,

$$
C^{-1} \leq\left|D\left(g_{n} ; z\right) / \sqrt{Q^{\prime}\left(a_{n}\right)}\right| \leq C .
$$

$$
C_{1} \leq Q^{\prime}\left(a_{n}\right) / \frac{n}{a_{n}} \leq C_{2}
$$

Proof. (a) By (6) and the unboundedness of $Q^{\prime}$ at $\infty$,

$$
0 \leq \frac{Q^{\prime \prime}(x)}{Q^{\prime}(x)^{2}} \leq \frac{\beta}{x Q^{\prime}(x)} \rightarrow 0, \quad x \rightarrow \infty .
$$

(b) This is a special case of the Nikolskii inequalities. See, for example, [24, p. 94] or [15, p. 295].

(c) Now, if $v \geq u \geq 0$,

$$
\log \frac{Q^{\prime}(v)}{Q^{\prime}(u)}=\int_{u}^{v} \frac{Q^{\prime \prime}(t)}{Q^{\prime}(t)} d t \geq \int_{u}^{v} \frac{\alpha}{t} d t=\alpha \log \frac{v}{u},
$$

by (6). Similarly, we obtain an upper bound, and hence,

$$
\left(\frac{v}{u}\right)^{\alpha} \leq \frac{Q^{\prime}(v)}{Q^{\prime}(u)} \leq\left(\frac{v}{u}\right)^{\beta}, \quad 0<u<v .
$$

Then, for $t \in[-\pi, \pi]$,

$$
\begin{aligned}
\mid \log \left(g_{n}(t)\right) & -\log Q^{\prime}\left(a_{n}\right) \mid \\
& =\left|\log \left(\frac{1}{Q^{\prime}\left(a_{n}\right)}+\left|\frac{Q^{\prime}\left(a_{n} \cos t\right)}{Q^{\prime}\left(a_{n}\right)}\right|\right)\right| \\
& \leq \max \left\{\log \left(\frac{1}{Q^{\prime}\left(a_{1}\right)}+1\right),\left.|\log | \cos t\right|^{\beta} \mid\right\} .
\end{aligned}
$$


Then, for $|z| \leq r<1$,

$$
\begin{aligned}
|\log | D\left(g_{n} ; z\right) / \sqrt{Q^{\prime}\left(a_{n}\right)}|| \\
=\left|\frac{1}{4 \pi} \int_{-\pi}^{\pi}\left[\log g_{n}(t)-\log Q^{\prime}\left(a_{n}\right)\right] \operatorname{Re}\left(\frac{e^{i t}+z}{e^{i t}-z}\right) d t\right| \\
\leq \frac{1}{4 \pi} \frac{1+r}{1-r} \int_{-\pi}^{\pi} \max \left\{\log \left(\frac{1}{Q^{\prime}\left(a_{1}\right)}+1\right),\left.|\log | \cos t\right|^{\beta} \mid\right\} d t .
\end{aligned}
$$

(d) As $Q^{\prime}$ is increasing,

$$
n=\frac{2}{\pi} \int_{0}^{1} \frac{a_{n} t Q^{\prime}\left(a_{n} t\right)}{\sqrt{1-t^{2}}} d t \leq a_{n} Q^{\prime}\left(a_{n}\right) .
$$

Also,

$$
n \geq \frac{2}{\pi} \int_{1 / 2}^{1} \frac{\left(a_{n} / 2\right) Q^{\prime}\left(\left(a_{n} / 2\right)\right)}{\sqrt{1-t^{2}}} d t \geq a_{n} Q^{\prime}\left(a_{n}\right) \frac{1}{\pi} \int_{1 / 2}^{1} \frac{1}{\sqrt{1-t^{2}}} d t\left(\frac{1}{2}\right)^{1+\beta},
$$

by (87).

Proof of (7) of Theorem 1.1. The weight $W=\exp (-Q)$ is even, so the numbers $a_{ \pm n}$ become just $\pm a_{n}$, while $\delta_{n}=a_{n}$. We apply Theorem 1.5 and note that, from (75),

$$
\frac{\gamma_{n-1}}{\gamma_{n}} \leq 4 a_{n}
$$

while we may take as the number $\eta_{n}$ in the Jackson inequality (33),

$$
\eta_{n}=C \frac{a_{n}}{n} .
$$

See [24, p. 81] or [7]. Then (76) becomes

$$
\left|\left(\frac{\gamma_{n-1}\left(W^{2}\right)}{n \kappa_{n}}\right)^{2}-\lambda\right|=O\left(\frac{a_{n}}{n}\right)^{2},
$$

so, from (79),

$$
\int_{I}\left(q_{n}^{\prime}-n \frac{\kappa_{n}}{\gamma_{n-1}\left(W^{2}\right)} p_{n-1}\right)^{2} W^{2} \leq \frac{1}{\lambda}-\left(n \frac{\kappa_{n}}{\gamma_{n-1}\left(W^{2}\right)}\right)^{2}=O\left(\frac{a_{n}}{n}\right)^{2} .
$$

Then also,

$$
\left|\frac{n \kappa_{n}}{\gamma_{n-1}\left(W^{2}\right)}-\frac{1}{\sqrt{\lambda}}\right|=O\left(\frac{a_{n}}{n}\right)^{2}
$$

so (80) yields

$$
\int_{I}\left(q_{n}^{\prime}-\frac{1}{\sqrt{\lambda}} p_{n-1}\right)^{2} W^{2}=O\left(\frac{a_{n}}{n}\right)^{2} .
$$

Next, we estimate $q_{n}(0)$.

34 
Lemma 5.2. Assume the hypotheses of Theorem 1.1. Then

$$
\left|q_{n}(0)\right| \leq C \sqrt{\frac{a_{n}}{n}} .
$$

Proof. First let us denote the Sobolev orthogonal polynomial for the special case $\psi \equiv 1$ by $q_{n}^{*}$. By Lemma 5.1(b),

$$
\left|q_{n}^{*} W\right|(0) \leq C \sqrt{\frac{n}{a_{n}}}\left\|q_{n}^{*} W\right\|_{L_{2}(\mathbf{R})} \leq C \sqrt{\frac{a_{n}}{n}},
$$

by (78), with $\psi \equiv 1$ and the estimates (88) above. Now we return to general $\psi$, denoting as usual the corresponding Sobolev orthogonal polynomials by $q_{n}$. The triangle inequality gives

$$
\begin{aligned}
\left|q_{n}(0)-q_{n}^{*}(0)\right|\|\psi W\|_{L_{2}(\mathbf{R}) \leq} \leq & \left\|\left(q_{n}-q_{n}^{*}\right) \psi W\right\|_{L_{2}(\mathbf{R})} \\
& +\left\|\left[\left(q_{n}-q_{n}^{*}\right)-\left(q_{n}-q_{n}^{*}\right)(0)\right] \psi W\right\|_{L_{2}(\mathbf{R}) .}
\end{aligned}
$$

Here by (78) and (88) applied to $q_{n}$ and $q_{n}^{*}$,

$$
\left\|\left(q_{n}-q_{n}^{*}\right) \psi W\right\|_{L_{2}(\mathbf{R})} \leq\left\|q_{n} \psi W\right\|_{L_{2}(\mathbf{R})}+\left\|q_{n}^{*} \psi W\right\|_{L_{2}(\mathbf{R})} \leq C \frac{a_{n}}{n} .
$$

Next, by Lemma 3.4(c),

$$
\begin{aligned}
\|\left[\left(q_{n}-q_{n}^{*}\right)\right. & \left.-\left(q_{n}-q_{n}^{*}\right)(0)\right] \psi W \|_{L_{2}(\mathbf{R})} \\
& \leq\|\psi\|_{L_{\infty}(\mathbf{R})} C_{2}\left\|\left(q_{n}-q_{n}^{*}\right)^{\prime} W\right\|_{L_{2}(\mathbf{R})} \\
& \leq C_{2}\|\psi\|_{L_{\infty}(\mathbf{R})}\left\|\left[\left(q_{n}^{\prime}-\frac{1}{\sqrt{\lambda}} p_{n-1}\right)-\left(q_{n}^{* \prime}-\frac{1}{\sqrt{\lambda}} p_{n-1}\right)\right] W\right\|_{L_{2}(\mathbf{R})} \\
& \leq C \frac{a_{n}}{n},
\end{aligned}
$$

by (7) applied to $q_{n}$ and $q_{n}^{*}$. Combining the last inequality and (91), (92) gives

$$
\left|q_{n}(0)-q_{n}^{*}(0)\right| \leq C \frac{a_{n}}{n} .
$$

Then (90) gives the result.

Proof of (8). This follows from what we have just proved, (7), and Lemma 3.4(c). More precisely, Lemma 3.4(c) gives (recall that (58) is valid by Lemma 5.1 above)

$$
\left\|\left(1+\left|Q^{\prime}\right|\right)\left(q_{n}-q_{n}(0)-\frac{1}{\sqrt{\lambda}} \int_{0}^{x} p_{n-1}\right) W\right\|_{L_{2}(\mathbf{R})}=O\left(\frac{a_{n}}{n}\right) .
$$

By Lemma 5.2,

$$
\left|q_{n}(0)\right|\left\|\left(1+\left|Q^{\prime}\right|\right) W\right\|_{L_{2}(\mathbf{R})} \leq C \sqrt{\frac{a_{n}}{n}} .
$$

Note that Lemma 5.1(a) implies that for each $\varepsilon>0, Q^{\prime 2} W^{\varepsilon}$ is decreasing for large $x$, so the norm in the last left-hand side is finite. Then (8) follows. 
Proof of (9) and (10). The Nikolskii inequality in Lemma 5.1(b) and (7) gives

$$
\left\|\left(q_{n}^{\prime}-\frac{1}{\sqrt{\lambda}} p_{n-1}\right) W\right\|_{L_{\infty}(\mathbf{R})} \leq C \sqrt{\frac{n}{a_{n}}}\left\|\left(q_{n}^{\prime}-\frac{1}{\sqrt{\lambda}} p_{n-1}\right) W\right\|_{L_{2}(\mathbf{R})} \leq C \sqrt{\frac{a_{n}}{n}} .
$$

Then Lemma 3.4 gives

$$
\left\|\left(1+\left|Q^{\prime}\right|\right)\left(q_{n}-q_{n}(0)-\frac{1}{\sqrt{\lambda}} \int_{0}^{x} p_{n-1}\right) W\right\|_{L_{\infty}(\mathbf{R})}=O\left(\sqrt{\frac{a_{n}}{n}}\right) .
$$

Our estimate above for $q_{n}(0)$ then gives the result.

Proof of (12) and (13). First, (12) follows from (7), (81), and the fact that $L_{n}^{[-1]}(z)=$ $a_{n} z$ in this even case. For (13), we use (82) and (94) to obtain, uniformly for $z$ in closed subsets of $\mathbf{C} \backslash[-1,1]$,

$$
\begin{aligned}
& \left|\frac{q_{n}\left(a_{n} z\right)-q_{n}(0)-(1 / \sqrt{\lambda}) \int_{0}^{a_{n} z} p_{n-1}}{\varphi(z)^{n} D^{-2}\left(W_{n}^{*} ; 1 / \varphi(z)\right)}\right|\left(1-|\varphi(z)|^{-1}\right) \\
& \quad \leq \pi^{-1 / 2} a_{n}^{-1 / 2}\left\|\left(\left|Q^{\prime}\right|+1\right)\left(q_{n}-q_{n}(0)-\frac{1}{\sqrt{\lambda}} \int_{0}^{x} p_{n-1}\right) W\right\|_{L_{2}\left[a_{-n}, a_{n}\right]} \\
& \quad=O\left(\frac{\sqrt{a_{n}}}{n}\right) .
\end{aligned}
$$

Here by (26) and Lemma 5.1(c), (d),

$$
\frac{n}{a_{n}}\left|D^{-2}\left(W_{n}^{*} ; \frac{1}{\varphi(z)}\right) / D^{-2}\left(W_{n} ; \frac{1}{\varphi(z)}\right)\right|=\frac{n}{a_{n}}\left|D^{-2}\left(g_{n} ; \frac{1}{\varphi(z)}\right)\right|
$$

is bounded above and below for $z$ in closed subsets of $\mathbf{C} \backslash[-1,1]$. Also, as $\log W_{n} \leq 0$,

$$
\left|D^{2}\left(W_{n} ; u\right)\right| \leq 1, \quad|u|<1 .
$$

Hence the term

$$
\left|q_{n}(0) /\left(\varphi(z)^{n} D^{-2}\left(W_{n}^{*} ; \frac{1}{\varphi(z)}\right)\right)\right| \leq C\left|q_{n}(0)\right| \frac{n}{a_{n}}\left|D^{2}\left(W_{n} ; \frac{1}{\varphi(z)}\right)\right| /|\varphi(z)|^{n}
$$

decays geometrically, in view of the decay (89) of $q_{n}(0)$, and the geometric growth of the denominator. We can then drop $q_{n}(0)$ in (95) and (13) follows.

Proof of Corollary 1.2. From [15, Theorem 15.1, p. 402],

$$
\gamma_{n-1}\left(W^{2}\right)=\frac{1}{\sqrt{2 \pi}}\left(\frac{a_{n}}{2}\right)^{-n+1 / 2} \exp \left(\frac{2}{\pi} \int_{0}^{a_{n}} \frac{Q(s)}{\sqrt{a_{n}^{2}-s^{2}}} d s\right)(1+o(1)) .
$$

Then (76) gives the result. Similarly, the $L_{2}$ asymptotics (15) follow directly from (7) of Theorem 1.1 and the $L_{2}$ asymptotics for $p_{n-1}$ given in [15, Theorem 15.1, p. 402]. The asymptotics in the plane (16) follow from (12) and asymptotics in the plane for $p_{n-1}$ given in [15, Theorem 15.1, p. 402]. Finally the pointwise asymptotics on the segment $\left[-a_{n}, a_{n}\right]$ follow from (9) and Theorem 15.3 in [15, p. 403]. (We warn the reader that the notation used there is a little different.) 
Acknowledgments. The work by F. Marcellan has been supported by Dirección General de Investigación (Ministerio de Ciencia y Technología) of Spain under grant BFM 2003-06335-C03-07, as well as NATO Collaborative grant PST.CLG 979738. J. Geronimo and D. Lubinsky, respectively, acknowledge support by NSF grants DMS-0200219 and DMS-0400446.

The authors would like to thank a referee for pointing out an omission in the proof of (8).

\section{References}

1. M. Alfaro, J. J. Moreno-Balcazar, T. E. Perez, M. A. Piñar, M. L. Rezola (2001): Asymptotics of Sobolev orthogonal polynomials for Hermite coherent pairs. J. Comput. Appl. Math., 133:141-150.

2. C. BennetT, R. SHARPLEY (1988): Interpolation of Operators. New York: Academic Press.

3. A. CACHAFEIRo, F. MARCELlan, J. J. Moreno-BALCAZAR (2003): On asymptotic properties of FreudSobolev orthogonal polynomials. J. Approx. Theory, 125:26-41.

4. P. DeIFT (1999): Orthogonal Polynomials and Random Matrices: A Riemann-Hilbert Approach. Courant Lecture Notes Math., Vol. 3. New York: Courant Institute.

5. P. Deift, T. KRiecherbauer, K. MCLaUghlin, S. Venakides, X. Zhou (1999): Strong asymptotics of orthogonal polynomials with respect to exponential weights. Comm. Pure Appl. Math., 52:1491-1552.

6. R. A. DeVore, G. G. LoRENTZ (1993): Constructive Approximation. Berlin: Springer-Verlag.

7. Z. DITZIAN, D. S. LUBINSKY (1997): Jackson and smoothness theorems for Freud weights in $L_{p}(0<$ $p \leq \infty)$. Constr. Approx., 13:99-152.

8. Z. DitZian, V. TOTIK (1987): Moduli of Smoothness. New York: Springer-Verlag.

9. A. J. DURAN (ED.) (1998): Proceedings of the VIIIth Symposium on Orthogonal Polynomials and Their Applications, Seville, Sept. 22-26, 1997 (A. J. Duran, ed.). J. Comput. Appl. Math., 99(1-2):1-533.

10. G. FreUd (1971): Orthogonal Polynomials. Budapest: Akademiai Kiado/Pergamon Press.

11. W. GAUTSCHI, A. B. J. KUIJLAARS (1997): Zeros and critical points of Sobolev orthogonal polynomials. J. Approx. Theory, 91:117-137.

12. J. S. GERONIMO, W. VAN ASSCHE (1990): Relative asymptotics for orthogonal polynomials with unbounded recurrence coefficients. J. Approx. Theory, 62:47-69.

13. A. ISERLES, P. E. KoCH, S. P. NORSETT, J. M. SANZ-SERNA (1991): On polynomials orthogonal with respect to certain Sobolev inner products. J. Approx. Theory, 65:151-175.

14. T. KRIECHERBAUER, K. T-R MCLAUGHLIN (1999): Strong asymptotics of polynomials orthogonal with respect to Freud weights. Int. Math. Res. Not., 6:299-333.

15. E. Levin, D. S. LuBINSKY (2001): Orthogonal Polynomials for Exponential Weights. New York: Springer-Verlag.

16. G. L. LOPEZ, F. MARCELLAN, W. VAN ASSCHE (1995): Relative asymptotics for polynomials orthogonal with respect to a discrete Sobolev inner product. Constr. Approx., 11:107-137.

17. G. G. LORENTZ, M. vON GolitsCheK, Y. MAKOvOZ (1996): Constructive Approximation: Advanced Problems. Berlin: Springer-Verlag.

18. D. S. LUBINSKY (2000): Asymptotics of orthogonal polynomials: Some old, some new, some identities. Acta Appl. Math., 61:207-256.

19. F. Marcellan, M. Alfaro, M. L. Rezola (1993): Orthogonal polynomials on Sobolev spaces: Old and new directions. J. Comput. Appl. Math., 48:113-131.

20. F. MARCELlan, J. J. Moreno-BalCAZAR (2001): Strong and Plancherel-Rotach asymptotics of nondiagonal Laguerre-Sobolev orthogonal polynomials. J. Approx. Theory, 110:54-73.

21. A. MARTíNEZ-FINKELSHTEIN (2001): Analytic aspects of Sobolev orthogonality revisited. J. Comput. Appl. Math., 127:255-266.

22. A. MARTÍNEZ-FINKELSHTEIN (2000): A Bernstein-Szegö theorem for Sobolev orthogonal polynomials. Constr. Approx., 16:73-84.

23. A. MartíneZ-Finkelshtein, J. J. Moreno-BalcaZar, T. E. Perez, M. A. Piñar (1998): Asymptotics of Sobolev orthogonal polynomials for coherent pairs of measures. J. Approx. Theory, 92:280-293. 
24. H. N. MHASKAR (1996): Introduction to the Theory of Weighted Polynomial Approximation. Singapore: World Scientific.

25. H. N. MHASKAR, E. B. SAFF (1984): Extremal problems for polynomials with exponential weights. Trans. Amer. Math. Soc., 285:204-234.

26. H. N. MHASKAR, E. B. SAFF (1985): Where does the sup norm of a weighted polynomial live? Constr. Approx., 1:71-91.

27. H. N. MHASKAR, E. B. SAFF (1987): Where does the $L_{p}$ norm of a weighted polynomial live? Trans. Amer. Math. Soc., 303:109-124.

28. P. NeVAI (1986): Geza Freud, Orthogonal polynomials and Christoffel functions: A case study. J. Approx. Theory, 48:3-167.

29. P. NeVAI (ED.) (1990): Orthogonal Polynomials and Special Functions: Theory and Practice. NATO ASI Series, Vol. 294. Dordrecht: Kluwer Academic.

30. P. NeVAI, V. TotiK (1986): Weighted polynomial inequalities. Constr. Approx., 2:113-127.

31. E. A. RAKHMANOV (1984): On asymptotic properties of polynomials orthogonal on the real axis. Mat. USSR Sb., 47:155-193.

32. E. A. RAKHMANOV (1992): Strong asymptotics for orthogonal polynomials associated with exponential weights on $\mathbb{R}$. In: Methods of Approximation Theory in Complex Analysis and Mathematical Physics (A. A. Gonchar and E. B. Saff, eds.). Moscow: Nauka, pp. 71-97.

33. E. B. SAFF, V. TotiK (1997): Logarithmic Potentials with External Fields. Berlin: Springer-Verlag.

34. G. SzEgo (1975): Orthogonal Polynomials. Amer. Math. Soc. Colloq. Publ., Vol. 23. Providence, RI: American Mathematical Society.

J. S. Geronimo

School of Mathematics

Georgia Institute of Technology

Atlanta, GA 30332

USA

geronimo@math.gatech.edu

\section{S. Lubinsky}

School of Mathematics

Georgia Institute of Technology

Atlanta, GA 30332

USA

lubinsky@math.gatech.edu
F. Marcellan

Departamento de Matematicas

Universidad Carlos III de Madrid

Madrid

Spain

pacomarc@ing.uc3m.es 